\title{
Bayesian Image Reconstruction from Partial Image and Aliased Spectral Intensity Data
}

\author{
Shyamsunder Baskaran, Student Member, IEEE, and Rick P. Millane, Senior Member, IEEE
}

\begin{abstract}
An image reconstruction problem motivated by $\mathrm{x}$ ray fiber diffraction analysis is considered. The experimental data are sums of the squares of the amplitudes of particular sets of Fourier coefficients of the electron density, and a part of the electron density is known. The image reconstruction problem is to estimate the unknown part of the electron density, the "image." A Bayesian approach is taken in which a prior model for the image is based on the fact that it consists of atoms, i.e., the unknown electron density consists of separated, sharp peaks. Currently used heuristic methods are shown to correspond to certain maximum a posteriori estimates of the Fourier coefficients. An analytical solution for the Bayesian minimum mean-squareerror estimate is derived. Simulations show that the minimum mean-square-error estimate gives good results, even when there is considerable data loss, and out-performs the maximum $a$ posteriori estimates.
\end{abstract}

Index Terms - Bayesian, fiber diffraction, Fourier transform, image reconstruction, inverse problems, MAP, MMSE, phase retrieval, $x$-ray crystallography.

\section{INTRODUCTION}

$\mathbf{T}$ HIS paper addresses an image reconstruction problem, motivated by a problem in x-ray crystallography, in which one has available partial information in image space and in Fourier space. X-ray crystallography is a technique for determining the structures (i.e., the position of each constituent atom) of molecules from measurements of the intensities of diffracted x-rays [1], [2]. Since the electrons are concentrated around the atomic nuclei, the atomic positions can be inferred from the distribution of electrons, the electron density function, (if it is reconstructed at sufficient resolution) within the crystal. $\mathrm{X}$-ray crystallography is therefore an image reconstruction problem, the electron density function being the image. Since a crystal is three-dimensional (3-D), the image to be reconstructed is 3-D. A crystalline specimen of the molecule under study is irradiated with a monochromatic beam of $\mathrm{x}$ rays and the resulting diffraction (scattering) pattern measured [Fig. 1(a)]. A 3-D data set is built up by collecting diffraction patterns for different orientations of the crystal in the incident x-ray beam [1], [2]. The diffraction pattern cannot be focused to form an image because of the short wavelength $(\sim 1 \AA)$ of the $\mathrm{x}$-rays, so the diffraction data are processed numerically to produce an image. A crystalline specimen, in which all

Manuscript received May 18, 1998; revised November 11, 1998. This work was supported by the National Science Foundation under Grant DBI-9722862. The associate editor coordinating the review of this manuscript and approving it for publication was Prof. Helen Na.

The authors are with the Computational Science and Engineering Program, Purdue University, West Lafayette, IN 47907-1160 USA (e-mail: rmillane@purdue.edu).

Publisher Item Identifier S 1057-7149(99)07550-8. the molecules are identically oriented and regularly spaced, is required in order to obtain data with a usable signal-to-noise ratio (SNR). The diffraction of the $\mathrm{x}$-rays by the electrons in the molecules is weak, and the complex diffracted amplitude is equal to the (3-D) Fourier transform of the electron density in the crystal. The measurements yield the intensity of the diffracted x-rays, which provides the modulus of the complex amplitude, but not the phase. Since the phase must be known to reconstruct the electron density directly (by inverse Fourier transformation), the inverse problem constitutes a phase problem. Phase problems occur in several other scientific fields such as microscopy, radio engineering and astronomy, where one measures the amplitude of the Fourier transform of a quantity of interest [1], [3].

Despite the apparent difficulties posed by phase problems, an image (in two or more dimensions) is (almost always) uniquely determined by the amplitude of its Fourier transform [1], [3], [4]. Uniqueness relies upon the image having compact support, and the resulting analyticity of its Fourier transform in the complex plane [5]. Unique reconstruction therefore requires that the amplitude be measured effectively continuously in Fourier space. Under these circumstances, reasonably reliable reconstruction algorithms have been developed [1], [6]. These uniqueness properties do not apply to the crystallographic problem however, because the electron density within the crystalline specimen is periodic. The reason for this is as follows [1]. By the sampling theorem, the intensity of the Fourier transform (the measured diffraction pattern) of the electron density within the crystal is equal to the intensity of the Fourier transform of one period of the electron density, sampled at the Nyquist spacing for the complex amplitude. The bandwidth of the intensity is twice that of the complex amplitude (by the autocorrelation theorem for Fourier transforms) so that the former is sampled at twice its Nyquist spacing along each dimension. The continuous intensity function cannot therefore be reconstructed from the samples, so that analyticity and uniqueness cannot be invoked, and the crystallographic phase problem is therefore underdetermined in general. Various experimental and computational (algorithmic) methods are used for dealing with this problem. These all involve either measuring more data or imposing $a$ priori constraints on the electron density, or both, to make up for the inadequacy of the data. These methods are not relevant to the problem addressed in this paper and are not discussed further here.

The problem addressed in this paper is motivated by a particular crystallographic technique known as $x$-ray fiber diffraction analysis [7]-[9]. This is an important technique 


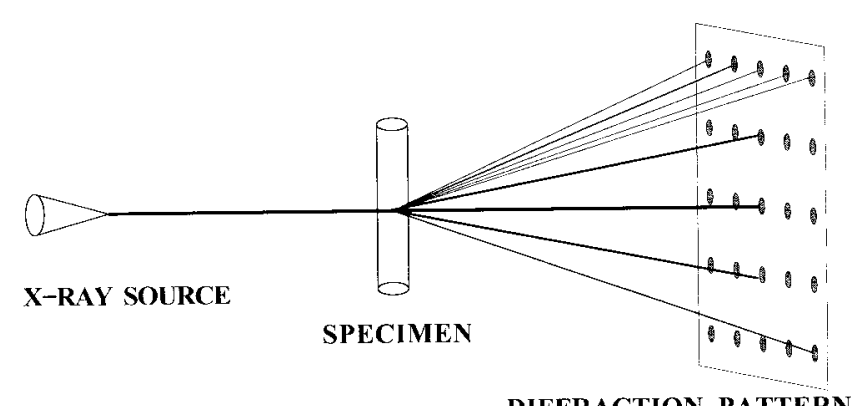

DIFFRACTION PATTERN

(a)

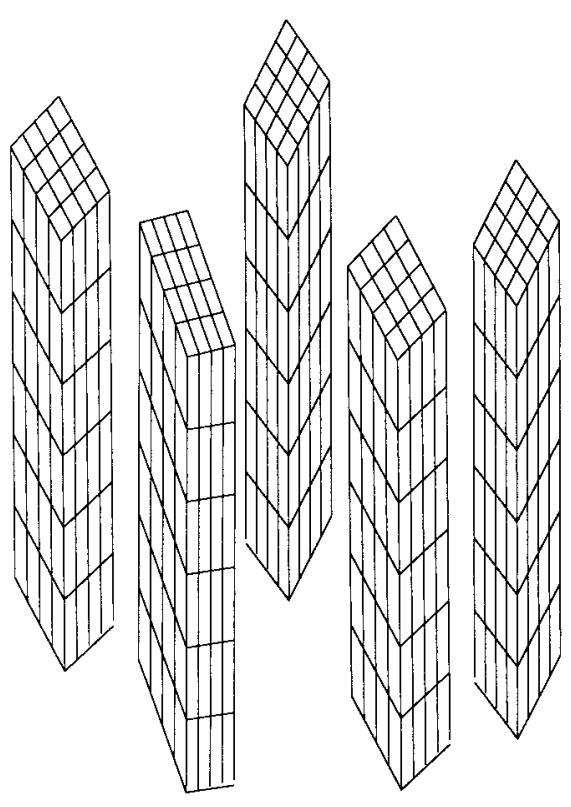

(b)

Fig. 1. (a) X-ray fiber diffraction experiment showing a beam of X-rays diffracted from the fiber specimen to form a diffraction pattern on a detector. (b) Schematic representation of a polycrystalline fiber, consisting of many small crystallites that are oriented parallel but are randomly rotated with respect to each other about the axis of orientation.

in materials science and structural biology that is used to determine the 3-D structures of synthetic and natural polymer molecules [7]. The information so obtained contributes to an understanding of the relationships between the structure and the physical and biological properties of such molecules, and is used as a guide for manipulating these properties. The most highly ordered polymer specimens that can be obtained for $\mathrm{x}$-ray analysis are called polycrystalline fibers. A polycrystalline fiber is made up of small crystallites, each of which has a unique axis. The crystallites are oriented so that these axes are parallel in the specimen, and each crystallite is randomly rotated about this axis [Fig. 1(b)]. Each crystallite diffracts independently, and so the measured diffraction pattern is the cylindrical average of the intensity diffracted by a single crystallite. Due to symmetry in the sampling lattice, the measured data are then sums of the squared amplitudes of sets of certain Fourier coefficients. Reconstruction of the electron density from such data, in the absence of additional information, is highly underdetermined. Solution methods primarily involve optimization of a model of the molecular structure (that incorporates many constraints) to minimize the mean-square difference between the data and the corresponding values calculated from the model [8], [9].

Two situations arise in fiber diffraction analysis where in addition to the diffraction data, one has also partial information on the structure (the electron density) [10]. The first situation is when part of the structure (the polymer) has been determined using a model as described above, and it remains to find the remainder, or the missing part (which cannot be incorporated into the initial model) of the structure. The missing structure often corresponds to counter-ions or solvent molecules, or sometimes mispositioned side-chains [11], [12]. The second situation occurs when the structure of a molecule that is known to be similar to the molecule under investigation is known, so that its electron density can be used as an initial approximation to help determine the unknown structure. In both cases, the problem of determining the unknown structure is equivalent to estimating the difference between a partial or approximate structure and the unknown structure. This is the image reconstruction problem addressed in this paper.

We consider the general problem of reconstruction of an image from data that are linear combinations of the squares of the amplitudes of sets of Fourier coefficients of the image (i.e., aliased spectral intensities), given partial information on the image. We adopt a Bayesian approach and determine explicit expressions for the minimum mean-square-error (MMSE) estimate, conditioned by an appropriate statistical model (a uniform distribution of atoms) for the missing part of the image. Methods currently used in x-ray fiber diffraction are analyzed and shown to correspond to certain maximum $a$ posteriori (MAP) estimates. Simulations are used to assess the performance of the MMSE estimate, and show its superiority over the MAP estimates. A preliminary account of this work has been reported [13].

The background for the particular crystallographic image reconstruction problem considered is presented in Section II, and a Bayesian framework for its solution is developed in Section III. The MMSE and MAP estimators are derived in Section IV, and their performances evaluated in Section V. The implications of these results are discussed in Section VI.

\section{BACKGROUND}

An x-ray diffraction experiment involves irradiating a crystalline specimen with a beam of monochromatic $x$-rays and recording the diffraction pattern. The electron density $\rho(\mathbf{r})$ in the crystal is periodic, i.e.

$$
\rho(\mathbf{r})=\varrho(\mathbf{r}) \otimes l(\mathbf{r})
$$

where $\mathbf{r}$ is position in the crystal, $\varrho(\mathbf{r})$ is the electron density in one period (a parallelepiped that is called the unit cell) and zero elsewhere, $\otimes$ denotes convolution, and $l(\mathbf{r})$ is the lattice

$$
l(\mathbf{r})=\sum_{(m, n, p) \in \mathbb{Z}^{3}} \delta(\mathbf{r}-m \mathbf{a}-n \mathbf{b}-p \mathbf{c})
$$

where $\mathbf{a}, \mathbf{b}$, and $\mathbf{c}$ are the basis vectors of the unit cell and $\mathbb{Z}$ is the set of integers. The complex diffracted amplitude, $F(\mathbf{u})$, 
is the Fourier transform of $\rho(\mathbf{r})$, so that

$$
F(\mathbf{u})=\mathcal{F}[\varrho(\mathbf{r})](\mathbf{u}) \times L(\mathbf{u})
$$

where $\mathcal{F}$ denotes the Fourier transform, $L(\mathbf{u})$ is the Fourier transform of $l(\mathbf{r})$ and $\mathbf{u}$ denotes position in Fourier or reciprocal space. Since $l(\mathbf{r})$ is periodic, $L(\mathbf{u})$ is a, generally oblique, periodic lattice in Fourier space called the reciprocal lattice. The observed intensity is $|F(\mathbf{u})|^{2}=\mid \mathcal{F}\left[\left.\varrho(\mathrm{r})\right|^{2}\right.$ sampled by $L(\mathbf{u})$. It is customary to denote the samples of $F(\mathbf{u})$ on $L(\mathbf{u})$ as $F_{\mathbf{h}}$, called the structure factors, where $\mathbf{h}=(h, k, l)$ is an integer triple that indexes the reciprocal lattice points, so that

$$
F_{\mathbf{h}}=\left|F_{\mathbf{h}}\right| \exp \left(i \phi_{\mathbf{h}}\right)=\int_{U} \varrho(\mathbf{r}) \exp (2 \pi i \mathbf{r} \cdot \mathbf{h}) d \mathbf{r}
$$

where $\left|F_{\mathbf{h}}\right|$ and $\phi_{\mathbf{h}}$ are the amplitude and phase of the structure factor, respectively, $U$ is the region inside the unit cell, and $d \mathrm{r}$ is the volume element in real space. The measured data are the intensities $\left|F_{\mathbf{h}}\right|^{2}$. The electron density function $\varrho(\mathbf{r})$ is related to the structure factors by inverse Fourier transformation, i.e.

$$
\varrho(\mathbf{r})=\frac{1}{V} \sum_{\mathbf{h}} F_{\mathbf{h}} \exp (-2 \pi i \mathbf{r} \cdot \mathbf{h})
$$

where $V$ is the volume of the region $U$. Sampling theory shows that the $F_{\mathbf{h}}$ are samples of $F(\mathbf{u})$ at Nyquist spacing. However, by virtue of the autocorrelation theorem for Fourier transforms [3], the bandwidth of $|F(\mathbf{u})|^{2}$ is twice that of $F(\mathbf{u})$, so that $\left|F_{\mathbf{h}}\right|^{2}$ samples the former at twice the Nyquist spacing along each coordinate of Fourier space.

Since the molecule is made up of atoms, and the electrons are crowded around the atomic nuclei, $\varrho(r)$ may be written in the form

$$
\varrho(\mathbf{r})=\sum_{j \in \mathcal{N}} \varrho_{j}\left(\mathbf{r}-\mathbf{r}_{j}\right)
$$

where $\varrho_{j}(\mathbf{r})$ is the electron density of the $j$ th atom when positioned at the origin, $\mathbf{r}_{j}$ its position, and the set $\mathcal{N}$ indexes the atoms in the unit cell. This property of the electron density function is called atomicity. The atomic number (the number of electrons) of the $j$ th atom, is given by $Z_{j}=\int \varrho_{j}(\mathbf{r}) d \mathbf{r}$. The Fourier transform relationship (4) may be written as a sum over the structure factors of the individual atoms, i.e.,

$$
\begin{aligned}
F_{\mathbf{h}} & =\sum_{j \in \mathcal{N}} \int_{U} \varrho_{j}\left(\mathbf{r}-\mathbf{r}_{j}\right) \exp (2 \pi i \mathbf{r} \cdot \mathbf{h}) d \mathbf{r} \\
& =\sum_{j \in \mathcal{N}} f_{j}(\mathbf{h}) \exp \left(i \phi_{j}(\mathbf{h})\right)
\end{aligned}
$$

where

$$
f_{j}(\mathbf{h})=\int \varrho_{j}(\mathbf{r}) \exp (2 \pi i \mathbf{r} \cdot \mathbf{h}) d \mathbf{r}
$$

and $\phi_{j}(\mathbf{h})=2 \pi \mathbf{r}_{j} \cdot \mathbf{h}$. The $f_{j}(\mathbf{h})$ are referred to as the atomic scattering factors. The $\phi_{j}(\mathbf{h})$ represent the phase shift resulting from the displacement of the atom from the origin. The dependence of the $f_{j}$ and $\phi_{j}$ on $\mathbf{h}$ is suppressed in the rest of this paper.

In the case of x-ray fiber diffraction analysis, the crystallites in the specimen are randomly rotated about the fiber axis
[Fig. 1(b)]. Since each crystallite diffracts independently, the observed data are the cylindrical average about the corresponding axis in reciprocal space, of the intensity diffracted by a single crystallite. Hence the data are linear combinations of the $\left|F_{\mathbf{h}}\right|^{2}$ that occur at reciprocal lattice points with the same (or very nearly the same) cylindrical polar radius in reciprocal space [8], [9]. The data $I_{j}$ may therefore be expressed as

$$
I_{j}=\sum_{\mathbf{h} \in \mathcal{S}_{j}}\left|F_{\mathbf{h}}\right|^{2}
$$

where the $\mathcal{S}_{j}$ are sets of reciprocal lattice points of the same cylindrical polar radius. We denote the set of structure factors belonging to the set $\mathcal{S}_{j}$ by $\left\{F_{\mathbf{h}}\right\}_{j}$, i.e. $\left\{F_{\mathbf{h}}\right\}_{j}=\left\{F_{\mathbf{h}}: \mathbf{h} \in\right.$ $\left.\mathcal{S}_{j}\right\}$. Clearly, this averaging results in a substantial reduction of the number of available diffraction data. The general image reconstruction problem in fiber diffraction then, is to reconstruct $\varrho(\mathbf{r})$ from the $I_{j}$. This corresponds to estimating the magnitudes and the phases of the structure factors $F_{\mathbf{h}}$ from each observed datum (followed by an inverse Fourier transformation). This may be visualized as a hyper-phase problem, wherein the hyper-angle representing the transformation from $I_{j}$ to $\left\{F_{\mathbf{h}}\right\}_{j}$ needs to be estimated, with the experimental datum providing the norm. The inverse problem is therefore underdetermined as posed. A similar problem occurs in x-ray powder diffraction where the reflections are spherically, rather than cylindrically, averaged and similar techniques have been used [14].

We examine in this paper the case where part of the structure $\varrho(r)$ is known, the practical importance of which has been described in the introduction. The electron density $\varrho(r)$ is represented as the sum of a known part, $\varrho^{P}(\mathbf{r})$, and an unknown or missing part, $\varrho^{Q}(\mathbf{r})$, i.e.

$$
\varrho(\mathbf{r})=\varrho^{P}(\mathbf{r})+\varrho^{Q}(\mathbf{r}) .
$$

The atoms belonging to $\varrho^{P}(\mathrm{r})$ and $\varrho^{Q}(\mathrm{r})$ are indexed by the sets $\mathcal{P}$ and $\mathcal{Q}$, respectively, so that $\mathcal{N}=\mathcal{P} \cup \mathcal{Q}$ and $\mathcal{P} \cap \mathcal{Q}=\emptyset$. It follows from (4) and (10) that

$$
F_{\mathbf{h}}=F_{\mathbf{h}}^{P}+F_{\mathbf{h}}^{Q}
$$

where $F_{\mathbf{h}}^{P}$ and $F_{\mathbf{h}}^{Q}$, respectively, are the structure factors of the known and missing parts. In summary, the problem is one of estimating $\varrho^{Q}(\mathrm{r})$, what we call here the image, from the intensity data $\left\{I_{j}\right\}$ and the known part of the image $\varrho^{P}(\mathbf{r})$.

Current approaches to this problem involve assigning approximate values $\hat{F}_{\mathbf{h}}$ to the structure factors $\left\{F_{\mathbf{h}}\right\}_{j}$ such that they satisfy (9), and using these to calculate $\varrho^{Q}(\mathbf{r})$ as $\mathcal{F}^{-1}\left[\hat{F}_{\mathbf{h}}-F_{\mathbf{h}}^{P}\right]$ where $\mathcal{F}^{-1}$ represents the inverse Fourier transform (5) [10]. In the first method, the estimates of $\left\{F_{\mathbf{h}}\right\}_{j}$, denoted by $\left\{\hat{F}_{\mathbf{h}}\right\}_{j}^{\mathrm{M} 1}$, are chosen so that the ratios of the $\left|\hat{F}_{\mathbf{h}}^{\mathrm{M} 1}\right|$ are the same as the ratios of the $\left|F_{\mathbf{h}}^{P}\right|$, and the phase of $\hat{F}_{\mathbf{h}}^{\mathrm{M} 1}$ is equal to the phase of $F_{\mathbf{h}}^{P}$, for $\mathbf{h} \in \mathcal{S}_{j}$. The estimate of the unknown structure factors is therefore given by

$$
\hat{F}_{\mathbf{h}}^{\mathrm{M} 1}=\sqrt{\frac{I_{j}}{I_{j}^{P}}} F_{\mathbf{h}}^{P}
$$

where $I_{j}^{P}=\Sigma_{\mathbf{h} \in \mathcal{S}_{j}}\left|F_{\mathbf{h}}^{P}\right|^{2}$. In the second method, the estimates of $\left\{F_{\mathbf{h}}\right\}_{j}$, denoted by $\left\{\hat{F}_{\mathbf{h}}\right\}_{j}^{\mathrm{M} 2}$, are chosen so that 
the $\left|\hat{F}_{\mathbf{h}}^{\mathrm{M} 2}\right|$ are all equal, and the phase of $\hat{F}_{\mathbf{h}}^{\mathrm{M} 2}$ is equal to the phase of $F_{\mathbf{h}}^{P}$. This gives

$$
\hat{F}_{\mathbf{h}}^{\mathrm{M} 2}=\sqrt{\frac{I_{j}}{\left|\mathcal{S}_{j}\right|}} \exp \left(i \phi_{\mathbf{h}}^{P}\right)
$$

where $|\cdot|$ denotes the number of elements in a set. Both methods have disadvantages. The former uses all the information on $\varrho^{P}(\mathrm{r})$ but the reconstruction may tend to contain features of $\varrho^{P}(\mathrm{r})$. The latter is parsimonious but does not fully utilize $\varrho^{P}(\mathrm{r})$. Both methods are used in practice [12], [15].

Our goal is to make optimal use of the data $I_{j}$ and $\varrho^{P}(\mathrm{r})$, as well as prior information on $\varrho^{Q}(\mathrm{r})$. This is analogous to methods in x-ray crystallography of single crystals where the individual $\left|F_{\mathbf{h}}\right|$, rather than the $I_{j}$, form the data [2]. We take a Bayesian approach to estimating $\varrho^{Q}(\mathrm{r})$, using a prior that is based on the atomicity property.

\section{StatisticAl FramewORK}

The models (stochastic or deterministic) for the image, transformation and observation that are used in the Bayesian formulation of the image reconstruction problem are presented in this section.

\section{A. Prior Model}

We use the atomicity property of $\varrho^{Q}(\mathbf{r})$ to define a prior for the image. In the absence of other information, the simplest choice for a prior model is one that consists of atoms whose positions are independent and are uniformly distributed within the unit cell. This model was first used by Wilson [16]. The drawback of this model is that it does not account for the dependence between atomic positions due to the finite size of the atoms, and for the propensity for certain geometric relationships between the atomic positions that result from the existence of particular chemical groups. These effects are extremely difficult to include in practice, and the simple model is often used and has found numerous applications in crystallography [17]. The prior density for the atomic positions is therefore

$$
\begin{aligned}
P_{\mathbf{r}_{j}}(\mathbf{r}) & =P_{x_{j}, y_{j}, z_{j}}(x, y, z) \\
& =(a b c)^{-1} \operatorname{rect}(x / a) \operatorname{rect}(y / b) \operatorname{rect}(z / c)
\end{aligned}
$$

where $\operatorname{rect}(x)=1$ for $|x|<1 / 2$ and zero for $|x|>1 / 2$.

The assumption that the positions of the atoms are independent implies that there are no symmetry relationships between groups of atoms in the unit cell. This corresponds to "space group P1" in crystallographic parlance [2], and is the case considered here. Symmetry relationships often exist between groups of atoms in the unit cell, in which case the estimation problem is more complicated and is considered elsewhere [18], [19].

\section{B. Transformation Model}

The prior model (14) applied to the image $\varrho^{Q}(\mathbf{r})$ allows a prior to be calculated for each Fourier coefficient $F_{\mathbf{h}}^{Q}$. Referring to (8), the phase factor $\phi_{j}$ for each atom is given by

$$
\phi_{j}=2 \pi \mathbf{r}_{j} \cdot \mathbf{h}=2 \pi\left(h x_{j} / a+k y_{j} / b+l z_{j} / c\right)
$$

and since $\phi_{j}$ is an angle, we may replace it with $\phi_{j} \bmod 2 \pi$. Referring to (14), the $\phi_{j}$ are then independent and uniformly distributed on $(-\pi, \pi)$, i.e.

$$
P_{\phi_{j}}(\phi)=(2 \pi)^{-1} \operatorname{rect}(\phi / 2 \pi) .
$$

The real part $\Re\left[F_{\mathbf{h}}^{Q}\right]=A_{\mathbf{h}}^{Q}=\Sigma_{j \in \mathcal{Q}} f_{j} \cos \left(\phi_{j}\right)$ and, referring to (16), $f_{j} \cos \left(\phi_{j}\right)$ is therefore distributed with zero mean and variance $f_{j}^{2}\left\langle\cos ^{2}\left(\phi_{j}\right)\right\rangle=f_{j}^{2} / 2$. Since the $\phi_{j}$ are independent, and assuming that the variance of the $f_{j}$ 's with respect to $j$ is small (i.e., the atoms are approximately of the same atomic number), the central limit theorem may be applied to (7) (provided that $|\mathcal{Q}|$ is large), so that

$$
P_{A_{\mathbf{h}}^{Q}}=\left(\pi \Sigma_{Q}\right)^{-1 / 2} \exp \left(-x^{2} / \Sigma_{Q}\right)
$$

where $\Sigma_{Q}$ is defined by

$$
\Sigma_{Q}=\sum_{j \in \mathcal{Q}} f_{j}^{2}
$$

The same distribution applies to $B_{\mathbf{h}}^{Q}=\Im\left[F_{\mathbf{h}}^{Q}\right]$. Equation (17)

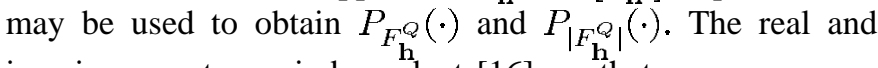
imaginary parts are independent [16], so that

$$
P_{F_{\mathbf{h}}^{Q}}(x+i y)=P_{A_{\mathbf{h}}^{Q}}(x) P_{B_{\mathbf{h}}^{Q}}(y) .
$$

$P_{F_{\mathbf{h}}^{Q}}(\cdot)$ is a function of the amplitude $\left|F_{\mathbf{h}}^{Q}\right|$ but is independent of the phase $\phi_{\mathbf{h}}^{Q}$, and $P_{\left|F_{\mathbf{h}}^{Q}\right|}(\cdot)$ may be obtained as

$$
P_{\left|F_{\mathbf{h}}^{Q}\right|}(\cdot)=2 x \Sigma_{Q}^{-1} \exp \left(-x^{2} / \Sigma_{Q}\right)
$$

Note that, referring to (8) and (18), $\Sigma_{Q}$ is a function of $\mathbf{h}$ in general. However, since the atomic electron densities are, to a very good approximation, spherically symmetric, so too are the atomic scattering factors $f_{j}(\mathbf{h})$ in reciprocal space. In x-ray fiber diffraction, the $\left\{F_{\mathbf{h}}\right\}_{j}$ for a given $j$ have the same cylindrical polar, and therefore the same spherical polar, radius in reciprocal space, and so $\Sigma_{Q}$ is fixed for a particular set $\mathcal{S}_{j}$.

\section{Observation Model}

Since the focus of this paper is on the effects of the partial information $I_{j}$ and $\varrho^{P}(\mathrm{r})$ on the estimation problem, we neglect measurement errors in the $I_{j}$ to simplify assessment of these effects. Referring to (9), the conditional density for the observation $I_{j}$ given $\left\{F_{\mathbf{h}}\right\}_{j}$ is therefore

$$
P_{I_{j} \mid\left\{F_{\mathbf{h}}\right\}_{j}}(\ell)=\frac{\Gamma\left(\left|\mathcal{S}_{j}\right|\right)}{2 \pi^{\left|\mathcal{S}_{j}\right| \ell\left|\mathcal{S}_{j}\right|-1 / 2}} \delta\left(\ell-\sum_{\mathbf{h} \in \mathcal{S}_{j}}\left|F_{\mathbf{h}}\right|^{2}\right)
$$

where $\Gamma(\cdot)$ is the gamma function. Since the $F_{\mathbf{h}}$ are Fourier coefficients, in the absence of prior information the components of $\left\{F_{\mathbf{h}}\right\}_{j}$ are independent and identically distributed, and the joint density for $\left\{F_{\mathbf{h}}\right\}_{j}$ is

$$
P_{\left\{F_{\mathbf{h}}\right\}_{j}}(\mathbf{y})=\prod_{\mathbf{h} \in \mathcal{S}_{j}} P_{F_{\mathbf{h}}}\left(y_{j}\right)
$$


where $\mathbf{y}=\left[y_{1}, y_{2}, \cdots\right]^{\dagger}$ is the column vector of $F_{\mathbf{h}}$ values and $^{\dagger}$ denotes transposition. Vectors of the real and imaginary parts of the structure factors in $\mathcal{S}_{j}$ are defined by

$$
\mathbf{Y}=\left[\begin{array}{c}
A_{h_{1}} \\
B_{h_{1}} \\
\vdots \\
B_{h_{n / 2}}
\end{array}\right], \quad \mathbf{X}=\left[\begin{array}{c}
A_{h_{1}}^{Q} \\
B_{h_{1}}^{Q} \\
\vdots \\
B_{h_{n / 2}}^{Q}
\end{array}\right] \quad \text { and } \quad \Theta=\left[\begin{array}{c}
A_{h_{1}}^{P} \\
B_{h_{1}}^{P} \\
\vdots \\
B_{h_{n / 2}}^{P}
\end{array}\right]
$$

where the subscript $j$ has been dropped, each vector has $n=2\left|\mathcal{S}_{j}\right|$ components, and $\mathbf{Y}=\mathbf{X}+\boldsymbol{\Theta}$. Equation (21) may then be written as

$$
P_{I_{j} \mid \mathbf{Y}}(\ell)=\frac{\Gamma(n / 2)}{2 \pi^{n / 2} \ell^{(n-1) / 2}} \delta\left(\ell-\|\mathbf{Y}\|^{2}\right)
$$

where $\|\mathbf{Y}\|$ is the Euclidean norm of $\mathbf{Y}$. From (17) and (22), the joint density for $\mathbf{X}$ may be written as [20], [21]

$$
P_{\mathbf{X}}(\mathrm{x})=\left(\pi \Sigma_{Q}\right)^{-n / 2} \exp \left(-\|\mathrm{x}\|^{2} / \Sigma_{Q}\right) .
$$

There is a common and fairly accurate assumption here that the prior distribution only weakly correlates the components of $\mathbf{X}$. Since $\boldsymbol{\Theta}$ is deterministic and $\mathbf{X}=\mathbf{Y}-\boldsymbol{\Theta}$, the joint density (22) may be written as

$$
P_{\mathbf{Y}}(\mathbf{y})=\left(\pi \Sigma_{Q}\right)^{-n / 2} \exp \left(-\|\mathbf{y}-\mathbf{\Theta}\|^{2} / \Sigma_{Q}\right) .
$$

\section{Posterior Distribution}

A comment on the notation is in order here. We assume that the data $I_{j}$ and the partial structure, expressed by $F_{\mathbf{h}}^{P}$, are both known exactly. In reality there will be errors associated with both of these quantities, but we ignore these effects for the rest of this paper. It is convenient however to refer to $I_{j}$ and $F_{\mathrm{h}}^{P}($ or $\Theta)$ as random variables (implicitly with zero variance) and to use notation such as $P_{\left\{F_{\mathbf{h}}\right\}_{j} \mid I_{j}}(\cdot)$ or $P_{\left\{F_{\mathbf{h}}\right\}_{j} \mid I_{j},\left\{F_{\mathbf{h}}^{P}\right\}_{j}}(\cdot)$. Although this is nonstandard notation, it is necessary so as to avoid ambiguity in the following, particularly for the MAP estimates.

The posterior distribution for the structure factors $\left\{F_{\mathbf{h}}\right\}_{j}$, given the intensity $I_{j}$, i.e., $P_{\mathbf{Y} \mid I_{j}}(\mathbf{y})$, is utilized in obtaining the Bayesian estimates in Section IV, and is derived here. (The reason for deriving $P_{\mathbf{Y} \mid I_{j}}(\mathbf{y})$ rather than $P_{\mathbf{X} \mid I_{j}}(\mathbf{x})$ is explained in Section IV.) Bayes theorem and the total probability theorem [22] may be applied to obtain the posterior distribution as

$$
P_{\mathbf{Y} \mid I_{j}}(\cdot)=\frac{P_{I_{j} \mid \mathbf{Y}}(\cdot) P_{\mathbf{Y}}(\cdot)}{P_{I_{j}}(\cdot)}=\frac{P_{I_{j} \mid \mathbf{Y}}(\cdot) P_{\mathbf{Y}}(\cdot)}{\int P_{I_{j} \mid \mathbf{Y}}(\mathbf{y}) P_{\mathbf{Y}}(\mathbf{y}) d \mathbf{y}} .
$$

Substituting using (24) and (26) gives

$$
P_{\mathbf{Y} \mid I_{j}}(\mathbf{y})=Z^{-1} \exp \left(-\|\mathbf{y}-\mathbf{\Theta}\|^{2} / \Sigma_{Q}\right) \delta\left(I_{j}-\|\mathbf{y}\|^{2}\right)
$$

where

$$
\begin{aligned}
Z & =\frac{2 \pi^{n} \sum_{Q}^{n / 2} I_{j}^{(n-1) / 2}}{\Gamma(n / 2)} \int P_{I_{j} \mid \mathbf{Y}}(\mathbf{y}) P_{\mathbf{Y}}(\mathbf{y}) d \mathbf{y} \\
& =\int \exp \left(-\|\mathbf{y}-\mathbf{\Theta}\|^{2} / \Sigma_{Q}\right) \delta\left(I_{j}-\|\mathbf{y}\|^{2}\right) d \mathbf{y}
\end{aligned}
$$

is the normalizing constant.
A simple analytical form for the posterior may be obtained by transforming (28) into a hyper-spherical polar coordinate system. Referring to Appendix $\mathrm{A},\|\mathbf{Y}\|=I_{j}^{1 / 2}$ is the radius, the polar axis is placed along $\boldsymbol{\Theta}$, and the first polar angle $\alpha_{1}$ is between $\mathbf{Y}$ and $\boldsymbol{\Theta}$. Equation (28) may then be expanded as

$$
\begin{aligned}
P_{\mathbf{Y} \mid I_{j}}(\mathbf{y})= & Z^{-1} \exp \left(-\left[\|\mathbf{y}\|^{2}+\|\mathbf{\Theta}\|^{2}-2\|\mathbf{y}\|\|\Theta\|\right.\right. \\
& \left.\cdot \cos \varphi] / \Sigma_{Q}\right) \delta\left(I_{j}-\|\mathbf{y}\|^{2}\right)
\end{aligned}
$$

where $\varphi \equiv \alpha_{1}$.

\section{BAYESIAN ESTIMATION}

Our objective is to estimate the image $\varrho^{Q}(\mathbf{r})$ using the intensity data $\left\{I_{j}\right\}$, the known part of the structure $\varrho^{P}(\mathbf{r})$, and the prior distribution for $\varrho^{Q}(\mathrm{r})$. Since $F_{\mathbf{h}}^{Q}$ and $F_{\mathbf{h}}^{P}$ are functions of only $\varrho^{Q}(\mathbf{r})$ and $\varrho^{P}(\mathbf{r})$, and since $F_{\mathbf{h}}=$ $F_{\mathbf{h}}^{P}+F_{\mathbf{h}}^{Q}$, where $F_{\mathbf{h}}^{P}$ is known, $F_{\mathbf{h}}$ can be estimated rather than $\varrho^{Q}(\mathbf{r})$. This gives simpler expressions for the estimates, and we therefore pose the problem in the following as that of estimating $F_{\mathbf{h}}$ given the $I_{j}$ and $F_{\mathbf{h}}^{P}$. The image, or the unknown electron density, $\varrho^{Q}(\mathbf{r})$, is then calculated as

$$
\hat{\varrho}^{Q}(\mathbf{r})=\mathcal{F}^{-1}\left[\hat{F}_{\mathbf{h}}-F_{\mathbf{h}}^{P}\right] .
$$

In the next section, a formal statistical basis for the currently used methods described in Section II is derived, and the MMSE estimate is derived in the following subsection.

\section{A. Currently Used Methods}

The currently used methods described in Section II correspond to well-defined statistical estimates of the structure factors, as is shown here.

Consider first the posterior density $P_{\left\{F_{\mathbf{h}}\right\}_{j} \mid I_{j},\left\{F_{\mathbf{h}}^{P}\right\}_{j}}(\cdot)$. The maximum of this density function corresponds to the maximum a posteriori estimate of the $\left\{F_{\mathbf{h}}\right\}_{j}$, given both the data $I_{j}$ and the known structure represented by $\left\{F_{\mathbf{h}}^{P}\right\}_{j}$, which we denote by $\left\{\hat{F}_{\mathbf{h}}\right\}_{j}^{\mathrm{MAP} 1}$, i.e.

$$
\left\{\hat{F}_{\mathbf{h}}\right\}_{j}^{\mathrm{MAP} 1}=\arg \max _{\mathbf{y}}\left[P_{\left\{F_{\mathbf{h}}\right\}_{j} \mid I_{j},\left\{F_{\mathbf{h}}^{P}\right\}_{j}}(\mathbf{y})\right]
$$

Using (30), the MAP1 estimate is given by

$$
\begin{aligned}
\hat{\mathbf{Y}}^{\mathrm{MAP} 1} & =\arg \max _{\mathbf{y}}\left[Z^{-1} \exp \left(-\|\mathbf{y}-\mathbf{\Theta}\|^{2} / \Sigma_{Q}\right) \delta\left(I_{j}-\|\mathbf{y}\|^{2}\right)\right] \\
& =\arg \min _{\mathbf{y}:\|\mathbf{y}\|=\sqrt{I_{j}}}\left[\|\mathbf{y}-\boldsymbol{\Theta}\|^{2}\right] \\
& =\arg \min _{\mathbf{y}:\|\mathbf{y}\|=\sqrt{I_{j}}}\left[\|\mathbf{y}\|^{2}+\|\boldsymbol{\Theta}\|^{2}-2\|\mathbf{y}\|\|\boldsymbol{\Theta}\| \cos \varphi\right]
\end{aligned}
$$

where $\varphi$ is the angle between $\mathbf{y}$ and $\boldsymbol{\Theta}$ [Fig. 2(a)]. Noting that $\|\mathbf{y}\|$ is fixed $\left(I_{j}^{1 / 2}\right)$ and $\Theta$ is known (deterministic), $\cos \varphi$ must be maximized in (33). Hence $\varphi=0$, i.e., $\mathbf{y}$ is parallel to $\Theta$ or $\hat{\mathbf{Y}}^{\mathrm{MAP} 1}=k \Theta$ where $k$ is a scalar. Therefore, the polar angles $\Omega_{\mathbf{y}}=\Omega_{\boldsymbol{\Theta}}$, and $\hat{\mathbf{Y}}^{\mathrm{MAP1}}=(\|\mathbf{y}\| /\|\Theta\|) \Theta$. In terms of 


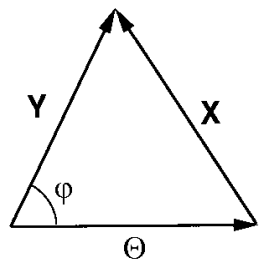

(a)

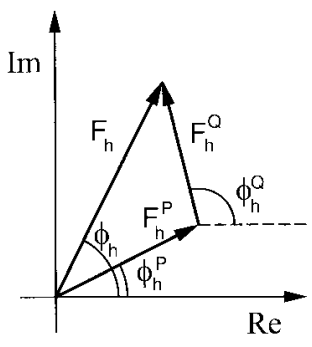

(b)

Fig. 2. (a) Relationship between $\mathbf{X}, \mathbf{Y}$, and $\Theta$. (b) Relationship between $F_{\mathbf{h}}, F_{\mathbf{h}}^{P}$, and $F_{\mathbf{h}}^{Q}$ in the complex plane.

the individual structure factors, the estimate is given by

$$
\hat{F}_{\mathbf{h}}^{\mathrm{MAP} 1}=\sqrt{\frac{I_{j}}{I_{j}^{P}}} F_{\mathbf{h}}^{P}, \quad \forall \mathbf{h} \in \mathcal{S}_{j} .
$$

Referring to (12), we see that $\hat{F}_{\mathbf{h}}^{\mathrm{M} 1}=\hat{F}_{\mathbf{h}}^{\mathrm{MAP} 1}$.

Consider now the marginal posterior density $P_{\left\{F_{\mathbf{h}}\right\}_{j} \mid I_{j},\left\{\phi_{\mathbf{h}}^{P}\right\}_{j}}(\cdot)$. Maximizing this density gives a MAP estimate of $F_{\mathbf{h}}$ that makes only partial use of the known structure, as only the phase, but not the amplitude, of the $F_{\mathrm{h}}^{P}$ are used. This should result in less tendency of the estimate toward the known part of the structure. We denote this estimate by $\left\{\hat{F}_{\mathbf{h}}\right\}_{j}^{\mathrm{MAP} 2}$ so that

$$
\begin{aligned}
\left\{\hat{F}_{\mathbf{h}}\right\}_{j}^{\mathrm{MAP} 2} & \\
& =\arg \max _{\mathbf{y}}\left[P_{\left\{F_{\mathbf{h}}\right\}_{j} \mid I_{j},\left\{\phi_{\mathbf{h}}^{P}\right\}_{j}}(\mathbf{y})\right] \\
& =\arg \max _{\{y\}_{j},\{\alpha\}_{j}}\left[P_{\left\{\left|F_{\mathbf{h}}\right|\right\}_{j},\left\{\phi_{\mathbf{h}}\right\}_{j} \mid I_{j},\left\{\phi_{\mathbf{h}}^{P}\right\}_{j}}\left(\{y\}_{j},\{\alpha\}_{j}\right)\right]
\end{aligned}
$$

where $F_{\mathbf{h}}$ has been separated into its amplitude and phase. The sets of random variables $\left\{\left\{\left|F_{\mathbf{h}}\right|\right\}_{j}, I_{j}\right\}$ and $\left\{\left\{\phi_{\mathbf{h}}\right\}_{j},\left\{\phi_{\mathbf{h}}^{P}\right\}_{j}\right\}$ are independent of each other, hence the density in (35) can be factored so that

$$
\begin{aligned}
& \left\{\hat{F}_{\mathbf{h}}\right\}_{j}^{\mathrm{MAP} 2} \\
& \quad=\arg \max _{\{y\}_{j},\{\alpha\}_{j}}\left[P_{\left\{\left|F_{\mathbf{h}}\right|\right\}_{j} \mid I_{j}}\left(\{y\}_{j}\right) P_{\left\{\phi_{\mathbf{h}}\right\}_{j} \mid\left\{\phi_{\mathbf{h}}^{P}\right\}_{j}}\left(\{\alpha\}_{j}\right)\right] .
\end{aligned}
$$

Therefore, the structure factor amplitudes and phases are given by

$$
\begin{aligned}
\left\{\left|\hat{F}_{\mathbf{h}}\right|\right\}_{j}^{\mathrm{MAP} 2} & =\arg \max _{\{y\}_{j}}\left[P_{\left\{\left|F_{\mathbf{h}}\right|\right\}_{j} \mid I_{j}}\left(\{y\}_{j}\right)\right] \\
\left\{\phi_{\mathbf{h}}\right\}_{j}^{\mathrm{MAP} 2} & =\arg \max _{\{\alpha\}_{j}}\left[P_{\left\{\phi_{\mathbf{h}}\right\}_{j} \mid\left\{\phi_{\mathbf{h}}^{P}\right\}_{j}}\left(\{\alpha\}_{j}\right)\right] .
\end{aligned}
$$

Using the density function (20) for the amplitudes gives

$$
\begin{aligned}
\left\{\left|\hat{F}_{\mathbf{h}}\right|\right\}_{j}^{\text {MAP2 }} & \arg \max _{\left\{y_{i}\right\}_{j}}\left[\left(\frac{2}{\Sigma_{N}}\right)^{n / 2}\left(\prod_{i=1}^{n / 2} y_{i}\right)\right. \\
& \left.\cdot \exp \left(-\left[\sum_{i=1}^{n / 2} y_{i}^{2}\right] / \Sigma_{N}\right) \delta\left(I_{j}-\sum_{i=1}^{n / 2} y_{i}^{2}\right)\right] \\
= & \arg \max _{\left\{y_{i}\right\}_{j}: \sum_{i=1}^{n / 2} y_{i}^{2}=I_{j}}\left[\prod_{i=1}^{n / 2} y_{i}\right] .
\end{aligned}
$$

The maximum of the product of the structure factor magnitudes under the constraint that the sum of their squares is a constant, occurs when they are all equal. The structure factor amplitudes are therefore given by

$$
\left|\hat{F}_{\mathbf{h}}\right|^{\mathrm{MAP} 2}=\sqrt{\frac{I_{j}}{\left|\mathcal{S}_{j}\right|}}, \quad \forall \mathbf{h} \in \mathcal{S}_{j} .
$$

Since $P_{\phi_{\mathbf{h}}}(\phi)=(2 \pi)^{-1} \operatorname{rect}(\phi / 2 \pi)$, and $\left|F_{\mathbf{h}}\right| \exp \left(i \phi_{\mathbf{h}}\right)=$ $\left|F_{\mathbf{h}}^{P}\right| \exp \left(i \phi_{\mathbf{h}}^{P}\right)+\left|F_{\mathbf{h}}^{Q}\right| \exp \left(i \phi_{\mathbf{h}}^{Q}\right)$, we have that [Fig. 2(b)]

$$
\begin{aligned}
P_{\phi_{\mathbf{h}} \mid \phi_{\mathbf{h}}^{P}}(\phi) \propto & \exp \left(-\left[\left|F_{\mathbf{h}}\right|^{2}+\left|F_{\mathbf{h}}^{P}\right|^{2}\right.\right. \\
& \left.\left.-2\left|F_{\mathbf{h}}\right|\left|F_{\mathbf{h}}^{P}\right| \cos \left(\phi_{\mathbf{h}}-\phi_{\mathbf{h}}^{P}\right)\right]\right)
\end{aligned}
$$

so that arg $\max _{\{\alpha\}_{j}}\left[P_{\left\{\phi_{\mathbf{h}}\right\}_{j} \mid\left\{\phi_{\mathbf{h}}^{P}\right\}_{j}}\left(\{\alpha\}_{j}\right)\right]=\left\{\phi_{\mathbf{h}}^{P}\right\}_{j}$, since $\left|F_{\mathbf{h}}\right|$ and $\left|F_{\mathbf{h}}^{P}\right|$ are fixed. The MAP2 structure factor estimates are therefore given by

$$
\hat{F}_{\mathbf{h}}^{\mathrm{MAP2}}=\sqrt{\frac{I_{j}}{\left|\mathcal{S}_{j}\right|}} \exp \left(i \phi_{\mathbf{h}}^{P}\right), \quad \forall \mathbf{h} \in \mathcal{S}_{j}
$$

and referring to (13) we see that $\hat{F}_{\mathbf{h}}^{\mathrm{M} 2}=\hat{F}_{\mathbf{h}}^{\mathrm{MAP} 2}$. Note that these two estimates maximize posterior densities for the Fourier coefficients, not the electron density.

\section{B. The MMSE Estimate}

Parseval's theorem [3], [22] shows that

$$
\int_{U}\left(\varrho^{Q}(\mathbf{r})-\hat{\varrho}^{Q}(\mathbf{r})\right)^{2} d \mathbf{r}=\sum_{\forall \mathbf{h}}\left|F_{\mathbf{h}}^{Q}-\hat{F}_{\mathbf{h}}^{Q}\right|^{2}
$$

i.e., an estimate of the electron density that minimizes the squared difference to the actual density, has Fourier coefficients that minimize the squared difference to the actual coefficients. The MMSE estimate for $F_{\mathbf{h}}^{Q}$ corresponds to minimizing $\|\mathbf{X}-\hat{\mathbf{X}}\|^{2}$ over $\hat{\mathbf{X}}$, and since $\boldsymbol{\Theta}$ is known, $\|\mathbf{X}-\hat{\mathbf{X}}\|^{2}=\|\mathbf{Y}-\hat{\mathbf{Y}}\|^{2}$, and we can minimize $\|\mathbf{Y}-\hat{\mathbf{Y}}\|^{2}$ over $\hat{\mathbf{Y}}$. The MMSE estimate of the missing electron density can therefore actually be obtained from the MMSE estimate of the $F_{\mathbf{h}}$ which is derived here.

The Bayesian MMSE estimate is (Appendix B)

$$
\hat{\mathbf{Y}}^{\mathrm{MMSE}}=\int \mathbf{y} P_{\mathbf{Y} \mid I_{j}}(\mathbf{y}) d \mathbf{y} .
$$

For the MMSE estimate, we make use of both the amplitude and phase information from $F_{\mathbf{h}}^{P}$ [i.e., $P_{\mathbf{Y} \mid I_{j}}(\mathbf{y})$ in (43) refers to 


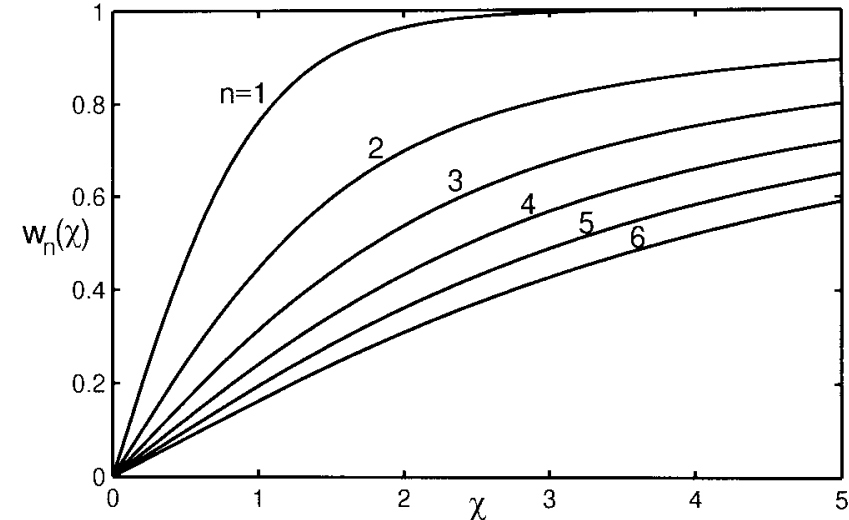

Fig. 3. Weighting function $w_{n}(\chi)$ as a function of $\chi$ for different values of $n$.

$\left.P_{\mathbf{Y} \mid I_{j}, \boldsymbol{\Theta}}(\mathbf{y})\right]$. The integrals in (29) and (43) can be evaluated (Appendix C), giving

$$
\hat{\mathbf{Y}}^{\mathrm{MMSE}}=\left(\|\hat{\mathbf{Y}}\|, \Omega_{\hat{\mathbf{Y}}}\right)^{\mathrm{MMSE}}=\left(\sqrt{I_{j}} \frac{I_{n / 2}(\chi)}{I_{n / 2-1}(\chi)}, \Omega_{\Theta}\right)
$$

where, referring to (62),

$$
\chi=\frac{2 \sqrt{I_{j} I_{j}^{P}}}{\Sigma_{Q}} .
$$

Hence the MMSE estimate $\varrho^{\mathrm{MMSE}}=\varrho^{Q \mathrm{MMSE}}+\varrho^{P}(\mathbf{r})$ is the inverse Fourier transform of the vector of Fourier coefficients $\hat{\mathbf{Y}}$ with norm $\sqrt{I_{j}} I_{n / 2}(\chi) / I_{n / 2-1}(\chi)$, and hyperphase $\Omega_{\mathbf{Y}}=\Omega_{\Theta} \equiv\left\{F_{\mathbf{h}}^{P}\right\}_{j} / \sqrt{I_{j}^{P}}$. The MMSE Fourier coefficients are therefore

$$
\hat{F}_{\mathbf{h}}^{\mathrm{MMSE}}=\frac{I_{n / 2}(\chi)}{I_{n / 2-1}(\chi)} \sqrt{\frac{I_{j}}{I_{j}^{P}}} F_{\mathbf{h}}^{P}, \quad \forall \mathbf{h} \in \mathcal{S}_{j} .
$$

The MMSE estimate of the image, i.e., the unknown electron density $\varrho^{Q}(\mathbf{x})$, is then calculated using $\hat{F}_{\mathbf{h}}^{\mathrm{MMSE}}$ for $\hat{F}_{\mathbf{h}}$ in $(31)$. Note that $\hat{F}_{\mathbf{h}}^{\mathrm{MMSE}}$ is a weight times $\hat{F}_{\mathbf{h}}^{\mathrm{MAP1}}$. The weighting function $w_{n}(\chi)=I_{n / 2}(\chi) / I_{n / 2-1}(\chi)$ reflects the uncertainty associated with the hyper-phase $\Omega_{\Theta}$ given the measured intensity, the known partial structure, and the overall amount of missing structure $\left(\Sigma_{Q}\right)$. A plot of the weighting function is shown in Fig. 3. The variable $\chi$ reflects the accuracy with which a particular $F_{\mathbf{h}}^{P}$ approximates $F_{\mathbf{h}}$, i.e., $\chi$ increases as $\Sigma_{Q}$ decreases (relative to $\sqrt{I_{j} I_{j}^{P}}$ ). Furthermore, the accuracy increases as $I_{j}^{P} \rightarrow I_{j}$, and since $I_{j}+I_{j}^{P}$ is fixed, $\sqrt{I_{j} I_{j}^{P}}$ increases, hence, so does $\chi$. As expected therefore, the weight $w_{n}(\chi)$ applied to a particular structure factor, increases with increasing $\chi$. The weight decreases with increasing $n$ since a larger $n$ represents less information on the individual structure factors within the datum $I_{j}$. The weights $w_{1}(\chi)$ and $w_{2}(\chi)$ correspond to those used in single crystal crystallography [23], [24].

\section{Parameter Estimation}

The quantity $\Sigma_{Q}$, given by (18), is a measure of the overall magnitude of the missing part of the electron density. This parameter must be estimated from the intensity data $\left\{I_{j}\right\}$ and the known part of the structure, represented by $F_{\mathrm{h}}^{P}$, in order to calculate the MMSE estimate derived above. Methods for estimating $\Sigma_{Q}$ for single crystal diffraction data have been reviewed by Read [25]. We derive here two estimates for $\Sigma_{Q}$ for the fiber diffraction case, based on those of Henderson and Moffat [26], and Nixon and North [27]. As noted in Section III-B, $\Sigma_{Q}$ is constant for a particular set $\mathcal{S}_{j}$, but in general varies with $j$.

For the first method, we consider the random walk from $\Theta$ to $\mathbf{Y}$ [26]. In $n_{j}=2\left|\mathcal{S}_{j}\right|$ dimensions, the expectation of the difference between the norms, $\left\langle\||\mathbf{Y}\|-\| \mathbf{\Theta} \||\rangle\right.$, is $1 / n_{j}$ times the expectation of the change in a single component, \langle|$Y_{i}-$ $\Theta_{i}|\rangle$, owing to the uncorrelated nature of the components. Since the expected difference in each component is given by $\left\langle\left|Y_{i}-\Theta_{i}\right|\right\rangle^{2}=\left\langle\left|X_{i}\right|\right\rangle^{2}=\Sigma_{Q}$, the first estimate $\hat{\Sigma}_{Q}^{\mathrm{M} 1}$ is

$$
\hat{\Sigma}_{Q}^{\mathrm{M} 1}=\left\langle n_{j}\left(\sqrt{I_{j}}-\sqrt{I_{j}^{P}}\right)^{2}\right\rangle_{j}
$$

where the averaging is over an ensemble of reflections indexed by $j$ for which $\Sigma_{Q}$ is assumed to be constant.

For the second method, we make use of the result of the statistical model described above [27]. Since $\hat{\mathbf{X}}=\hat{\mathbf{Y}}-\boldsymbol{\Theta}$, $\left\langle\|\hat{\mathbf{X}}\|^{2}\right\rangle=\|\hat{\mathbf{Y}}\|^{2}+\|\Theta\|^{2}-2\|\hat{\mathbf{Y}}\|\|\mathbf{\Theta}\| I_{n / 2}(\chi) / I_{n / 2-1}(\chi)$, and since $\chi=2 \sqrt{I_{j} I_{j}^{P}} / \Sigma_{Q}$, and $\left\langle\|\hat{\mathbf{X}}\|^{2}\right\rangle=\hat{\Sigma}_{Q}$, we obtain the second estimate as

$$
\hat{\Sigma}_{Q}^{\mathrm{M} 2}=\left\langle I_{j}+I_{j}^{P}-2\left(I_{n_{j} / 2}(\chi) / I_{n_{j} / 2-1}(\chi)\right) \sqrt{I_{j} I_{j}^{P}}\right\rangle_{j}
$$

which is an implicit equation for $\hat{\Sigma}_{Q}$, (since $\chi$ is a function of $\left.\Sigma_{Q}\right) . \hat{\Sigma}_{Q}^{\mathrm{M} 1}$ is substituted in the rhs of (48) to obtain the first estimate of $\hat{\Sigma}_{Q}^{\mathrm{M} 2}$. This value of $\hat{\Sigma}_{Q}^{\mathrm{M} 2}$ is substituted in the rhs (48) to obtain a new estimate, and the procedure repeated until convergence.

The uncertainty associated with the estimate from the datum $I_{j}$ increases with $n_{j} . \hat{\Sigma}_{Q}^{\mathrm{M} 1}$ tends to provide more weight for larger $n_{j}$ while $\hat{\Sigma}_{Q}^{\mathrm{M} 2}$ tends to weight each individual datum by its uncertainty. We therefore expect $\hat{\Sigma}_{Q}^{\mathrm{M} 2}$ to be more consistent than $\hat{\Sigma}_{Q}^{\mathrm{M} 1}$ in estimating $\Sigma_{Q}$. The accuracy of these two estimates is examined in the next section.

\section{Simulations}

In summary, we have derived an analytical solution for the Bayesian MMSE estimate and have shown that currently used methods correspond to the maxima of certain posterior densities. Computational experiments were used to compare the performance of these different estimates, and the results are presented in this section.

\section{A. Methods}

Although the crystallographic problem is $3-\mathrm{D}$, the results derived above apply to image reconstruction in any number of dimensions, and the simulations were performed on twodimensional (2-D) images. Since the structure factors $F_{\mathbf{h}}$ are 
at Nyquist spacing, the diffraction calculations are made by representing the electron density in the unit cell by a full (i.e., not zero-padded) $n_{x} \times n_{y}$ array and the $F_{\mathbf{h}}$ calculated as the discrete Fourier transform (DFT) (using the fast Fourier transform) of this array. The electron density consists of "atoms" (6), and for the simulations we used "point atoms" that are one pixel in size. The pixel value is then equal to the atomic number of the atom. Although real atoms are not sharp points, the resolution of typical fiber diffraction data is less than the spatial extent of the atoms so that the use of point atoms gives a good representation of the actual experiment. The electron densities $\varrho(\mathbf{r})$ for the simulations were produced by randomly placing $|\mathcal{N}|$ atoms in the array representing the unit cell, their atomic numbers being generated from a uniform distribution on $\left(Z_{\min }, Z_{\max }\right)$, and the structure factors $F_{\mathbf{h}}$ calculated using the fast Fourier transform.

In x-ray fiber diffraction, the set $\mathcal{S}_{j}$ consists of reciprocal lattice points with the same (or very nearly the same) cylindrical polar radii. The sets $\mathcal{S}_{j}$ are therefore determined by the symmetry of the reciprocal lattice. In the simulations, we used a more generic combination of the Fourier amplitudes so that the degree of aliasing, $\left\langle\left|\mathcal{S}_{j}\right|\right\rangle_{j}$, could be varied as desired. Fourier amplitudes were randomly combined into sets $\mathcal{S}_{j}$ to give the data $I_{j}$, such that $\left|\mathcal{S}_{j}\right|=1,2, \cdots, S_{\max }$, with an equal number of sets for each $\left|\mathcal{S}_{j}\right|$. The extent of data reduction is represented using $S_{\max }$, which was varied from 1-5. The data $I_{j}$ were calculated using (9).

Since point atoms were used for the simulations, $f_{j}$ is independent of $\mathbf{h}$, and $\Sigma_{Q}$ has the same value throughout reciprocal space, so that its value can be estimated using all the data $I_{j}$ and $I_{j}^{P}$, using (47) or (48). Therefore, use of point atoms and the random selection of structure factors (with random $\|\mathbf{h}\|)$ retains correspondence with the actual x-ray fiber diffraction experiment.

The known part of the structure $\varrho^{P}(\mathbf{r})$ was generated by removing $|\mathcal{Q}|$ randomly chosen atoms from $\varrho(\mathbf{r})$, and used to calculate the structure factors $F_{\mathbf{h}}^{P}$. The $\left\{\hat{F}_{\mathbf{h}}\right\}_{j}$ are then estimated from the information $\left\{F_{\mathbf{h}}^{P}\right\}_{j}$ and $I_{j}$, using the three different algorithms described in Section IV, and $\hat{\varrho}^{Q}(\mathbf{r})$ is calculated using equations of the form (31). The accuracy of the estimated image $\hat{\varrho}^{Q}(\mathbf{r})$ is assessed by comparing it to the true image using the correlation coefficient

$$
C=\frac{\int_{U} \hat{\varrho}^{Q}(\mathbf{r}) \varrho^{Q}(\mathbf{r}) d \mathbf{r}}{\left[\int_{U}\left(\hat{\varrho}^{Q}(\mathbf{r})\right)^{2} d \mathbf{r} \int_{U}\left(\varrho^{Q}(\mathbf{r})\right)^{2} d \mathbf{r}\right]^{1 / 2}} .
$$

For a perfect reconstruction $C=1$, and $C=0$ for a reconstruction that is not correlated with the true image. The estimators are studied as a function of two parameters; the amount of missing structure, quantified by $\Delta Q=$ $\Sigma_{j \in \mathcal{Q}} f_{j} / \Sigma_{j \in \mathcal{N}} f_{j}$, and the degree of data loss, quantified by $S_{\max }$. Since the atoms in $\mathcal{Q}$ and their atomic numbers are chosen randomly, $\Delta Q \approx|\mathcal{Q}| /|\mathcal{N}|$, which we use to calculate $\Delta Q$. Estimation of the parameter $\Sigma_{Q}$ is studied in the next subsection and the image reconstruction in the following section.

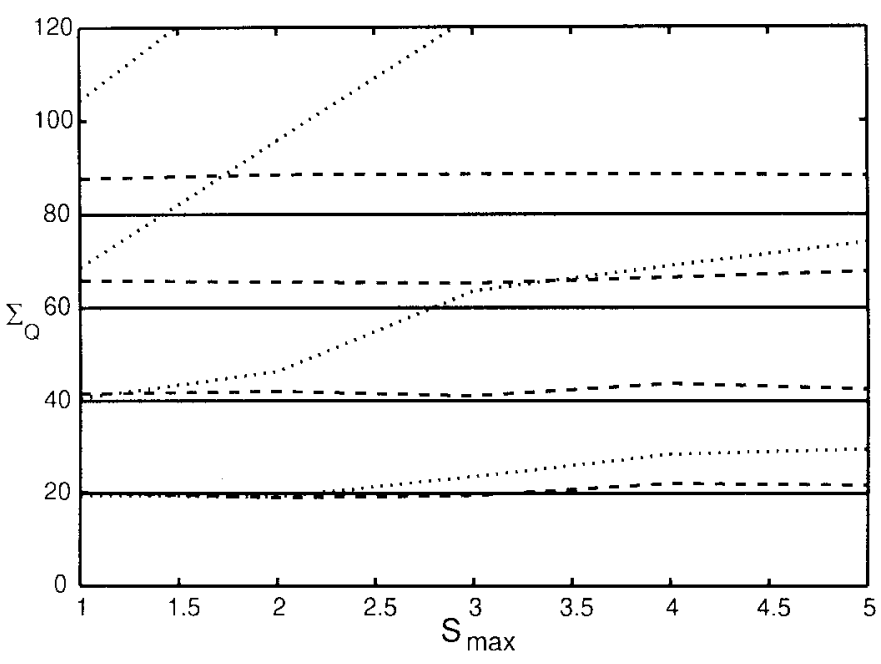

(a)

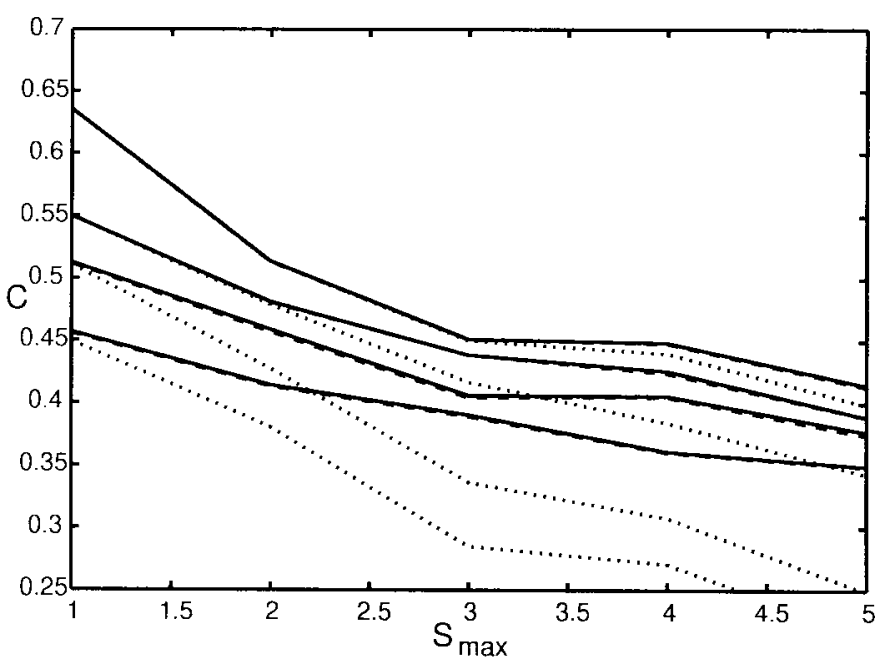

(b)

Fig. 4. (a) Estimates $\hat{\Sigma}_{Q}^{\mathrm{M} 1}(\ldots \ldots)$ and $\hat{\Sigma}_{Q}^{\mathrm{M} 2}(---)$, compared with the true value $\Sigma_{Q}$ (-), as a function of $S_{\max }$ for $\Delta Q=0.2,0.4,0.6$, and 0.8. (b) Correlation of the MMSE estimates obtained using $\hat{\Sigma}_{Q}^{\mathrm{M} 1}(\ldots \ldots)$, $\hat{\Sigma}_{Q}^{\mathrm{M} 2}(---)$, and $\Sigma_{Q}(-)$ in (a) as a function of $S_{\max }$ for the four values of $\triangle Q$.

\section{B. Estimation of $\Sigma_{Q}$}

The parameter $\Sigma_{Q}$ is needed to calculate the weighting function for the MMSE reconstruction as described in Section IV-C. The performance of the estimates $\hat{\Sigma}_{Q}^{\mathrm{M} 1}$ and $\hat{\Sigma}_{Q}^{\mathrm{M} 2}$ derived above was evaluated by simulation and the results are described here. Electron density functions were constructed and intensities $I_{j}$ calculated as described above. The estimates $\hat{\Sigma}_{Q}^{\mathrm{M} 1}$ and $\hat{\Sigma}_{Q}^{\mathrm{M} 2}$ were then calculated using (47) and (48), respectively. For the simulations, we used $n_{x}=n_{y}=32,|\mathcal{N}|=100$, and $Z_{\min }=Z_{\max }=1$, i.e., all the atoms have the same atomic number. The amount of missing structure $\Delta Q$ was varied as $\Delta Q=0.2,0.4,0.6$, and 0.8 , and the overlap $S_{\max }=1,2,3,4$, and 5 . The estimates $\hat{\Sigma}_{Q}^{\mathrm{M} 1}$ and $\hat{\Sigma}_{Q}^{\mathrm{M} 2}$ for the two methods are shown as a function of $S_{\max }$ for the four different values of $\Delta Q$ in Fig. 4(a). The estimate $\hat{\Sigma}_{Q}^{\mathrm{M} 2}$ is quite accurate, even for larger values of $S_{\max }$ 


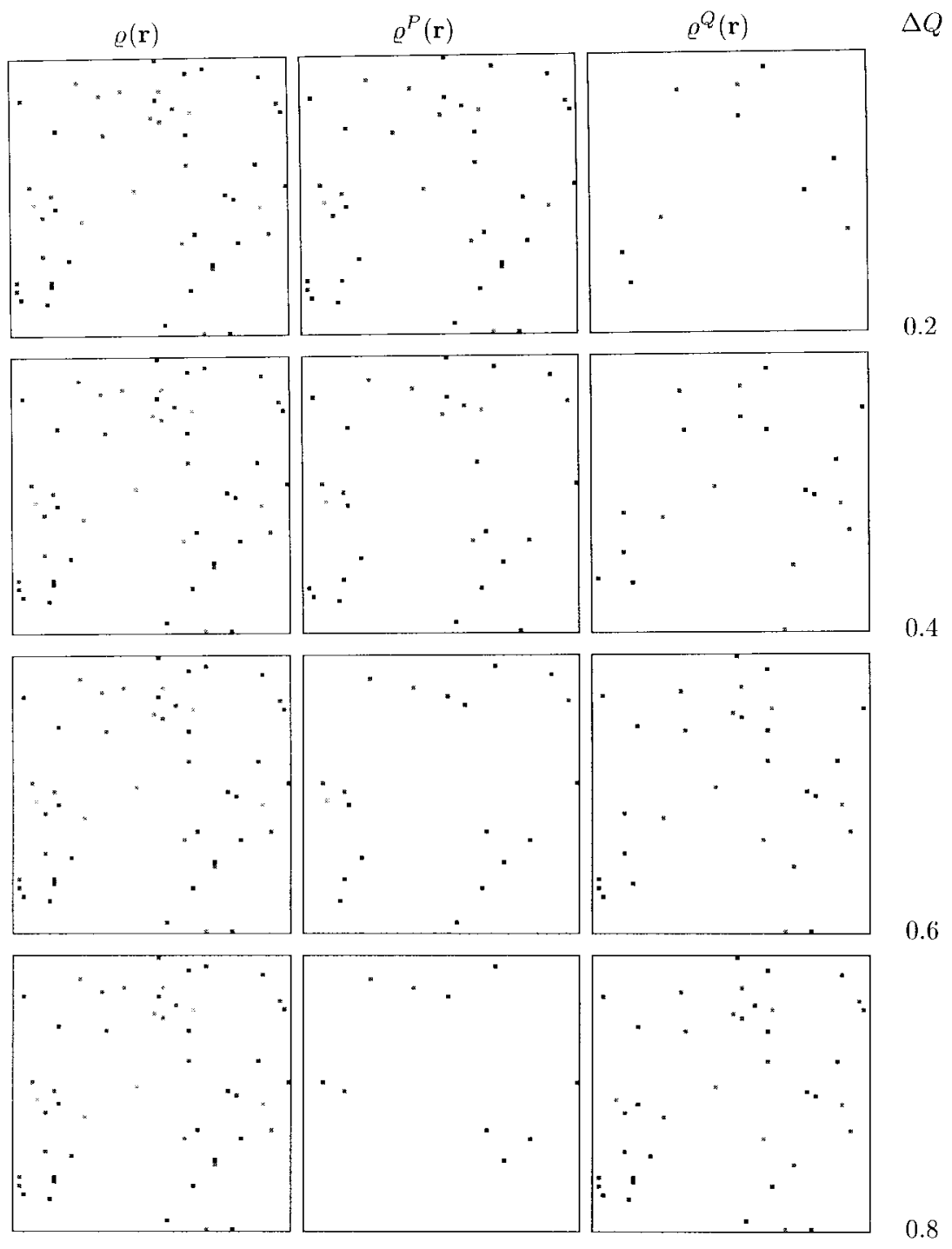

Fig. 5. True test images used in the simulations: $\varrho(\mathbf{r}), \varrho^{P}(\mathbf{r})$, and $\varrho^{Q}(\mathbf{r})$ for $\Delta Q=0.2,0.4,0.6$, and 0.8 .

and $\Delta Q$. The estimate $\hat{\Sigma}_{Q}^{\mathrm{M} 1}$ is accurate for smaller values of $S_{\max }$ and $\Delta Q$ but is very poor for larger values. The estimate $\hat{\Sigma}_{Q}^{\mathrm{M} 2}$ is therefore better than $\hat{\Sigma}_{Q}^{\mathrm{M} 1}$, as expected.

To assess the effects of errors in $\hat{\Sigma}_{Q}$ on the reconstructed image, $\hat{\varrho}^{Q}$ MMSE was calculated as described in Section IVB using values of $\chi$ calculated using the estimates $\hat{\Sigma}_{Q}^{\mathrm{M} 1}$ and $\hat{\Sigma}_{Q}^{\mathrm{M} 2}$, as well as using the true value $\Sigma_{Q}$. The accuracy of the resulting $\hat{\varrho}^{Q \mathrm{MMSE}}$ was calculated using the correlation coefficient (49) for the range of values of $\Delta Q$ and $S_{\max }$ used, and is shown in Fig. 4(b). Inspection of the figure shows that use of $\hat{\Sigma}_{Q}^{\mathrm{M} 2}$ gives essentially identical results to those obtained using the true value, for all values of $\Delta Q$ and $S_{\max }$ studied. Images reconstructed using $\hat{\Sigma}_{Q}^{\mathrm{M} 1}$ are less accurate for larger values of $\Delta Q$ and $S_{\max }$. Since $\hat{\Sigma}_{Q}^{\mathrm{M} 2}$ performs significantly better than $\hat{\Sigma}_{Q}^{\mathrm{M} 1}$ for higher values of $S_{\max }$ and $\Sigma_{Q}$, it was therefore used for the remaining simulations.

\section{Image Reconstruction}

In order to compare the performance of the MAP1, MAP2, and MMSE estimates described in Section IV, simulations were performed over a range of values of $\Delta Q$ and $S_{\max }$. The reconstructed images were assessed by calculating the correlation coefficient $C$ and by visual inspection. Test images were generated as described above with $n_{x}=n_{y}=64$, $Z_{\min }=25, Z_{\max }=50,|\mathcal{N}|=50, \Delta Q=0.2,0.4,0.6$, and 0.8 and $S_{\max }=1,2,3,4$, and 5 . The test images $\varrho(\mathbf{r}), \varrho^{P}(\mathbf{r})$ and $\varrho^{Q}(\mathbf{r})$ for the four values of $\Delta Q$ are shown in Fig. 5 .

In practice, the estimated electron density is used to locate the positions of the missing atoms. Hence, the amplitude of the electron density above the background, the sharpness of the peaks, and an absence of spurious peaks or artifacts, are used as qualitative characteristics of a good estimate. A poor estimate typically has a noisy background, weak "smeared out" peaks, and peaks at wrong locations, all of which hinder correct interpretability of the image.

The correlation coefficients between $\hat{\varrho}^{Q}(\mathbf{r})$ and $\varrho^{Q}(\mathbf{r})$ for the three different estimates, as a function of $\Delta Q$ and $S_{\max }$, are shown in Fig. 6. As expected, all estimates deteriorate with increasing $\Delta Q$ and increasing $S_{\max }$, since the known part becomes less representative of the image for the former, and there are fewer data $I_{j}$ for the latter. In all cases, the MMSE 


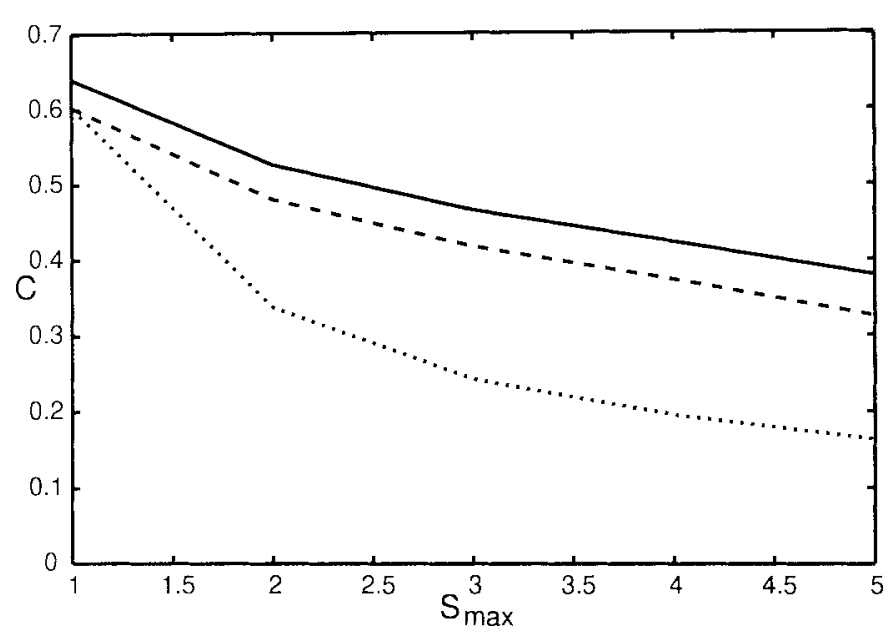

(a)

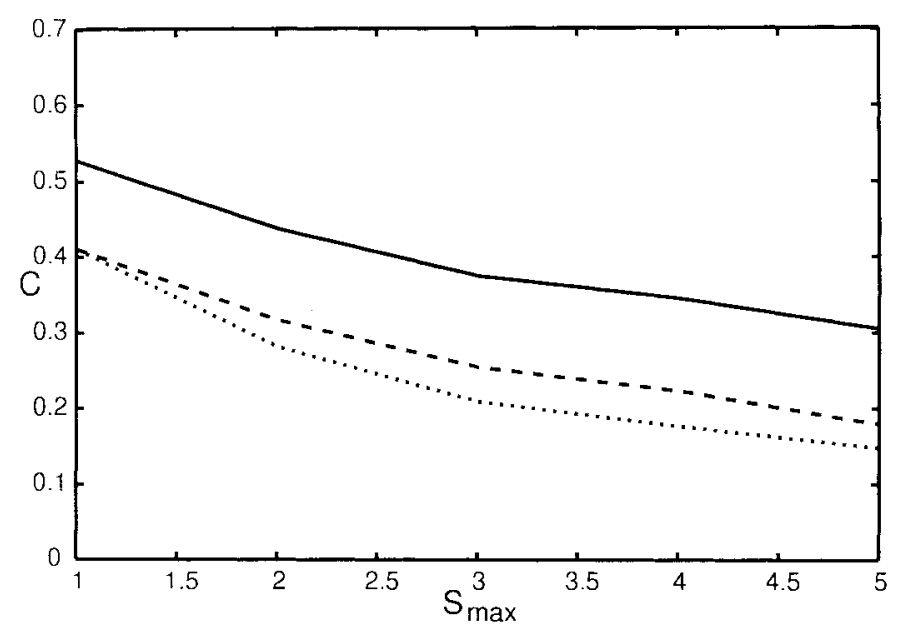

(c)

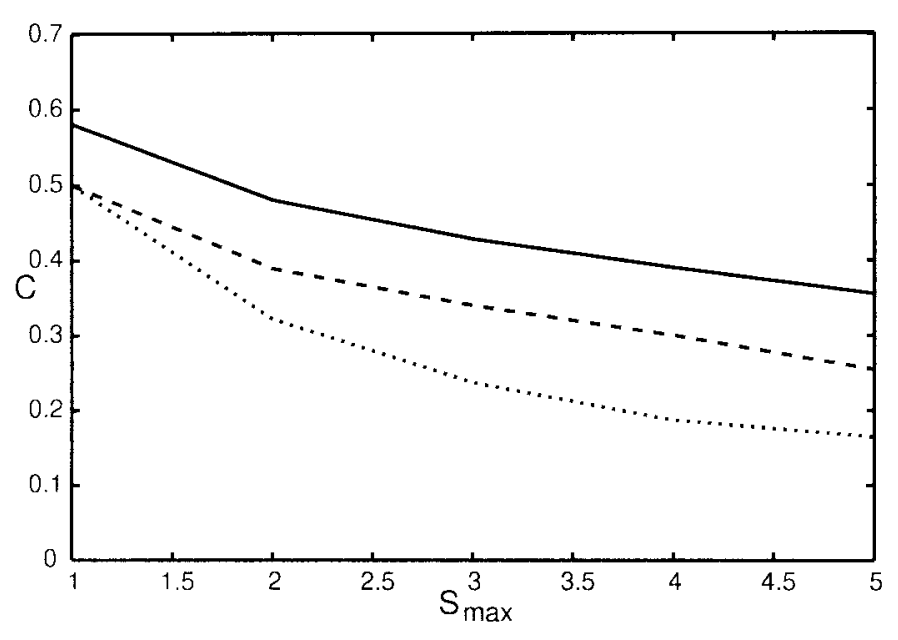

(b)

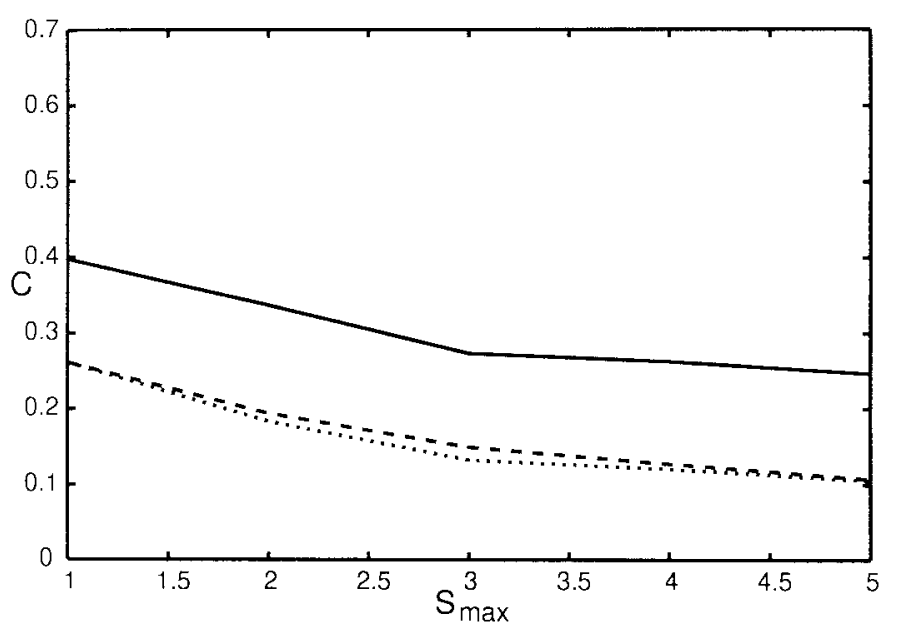

(d)

Fig. 6. Correlation coefficient between the true and estimated images for the MMSE (- - , MAP1 ( - - - ), and MAP2 ( $\ldots \ldots)$ estimates of $\varrho^{Q}(\mathbf{r})$, as a function of $S_{\max }$, for (a) $\Delta Q=0.2$, (b) $\Delta Q=0.4$, (c) $\Delta Q=0.6$, and (d) $\Delta Q=0.8$.

estimate performs the best and the MAP1 estimate the second best. When the missing part of the structure represents a small part of the total structure, the MAP1 estimate is almost as good as the MMSE estimate, while the MAP2 estimate is quite inferior. When larger amounts of the structure are missing, both the MAP estimates perform rather similarly, and the MMSE estimate is quite superior to both. The margin between the MAP1 and MAP2 estimates tends to increase with $S_{\max }$. These characteristics are also seen in Fig. 7 which shows the correlation coefficient as a function of $\Delta Q$ for $S_{\max }$ being 2 and 4 .

The true and estimated images for $S_{\max }=1,2,3,4$, and 5 are shown in Figs. 8-10 for $\Delta Q=0.2,0.4$, and 0.6, respectively. All images are displayed so that their minimum value is white and their maximum value is black. Inspection of Fig. $8(\Delta Q=0.2)$ shows that $\hat{\varrho}^{Q \text { MMSE }}$ is a very interpretable version of $\varrho^{Q}(\mathrm{r})$, for all values of $S_{\max }$. The MAP estimates are also good for small $S_{\max }$, but for $S_{\max }>3$ spurious peaks begin to appear, and the background noise increases in the MAP2 estimate. Some of the spurious peaks, in fact, correspond to peaks in the known part of the image, $\varrho^{P}(\mathbf{r})$ (see Fig. 5), since the reconstructions are dominated by $F_{\mathbf{h}}^{P}$

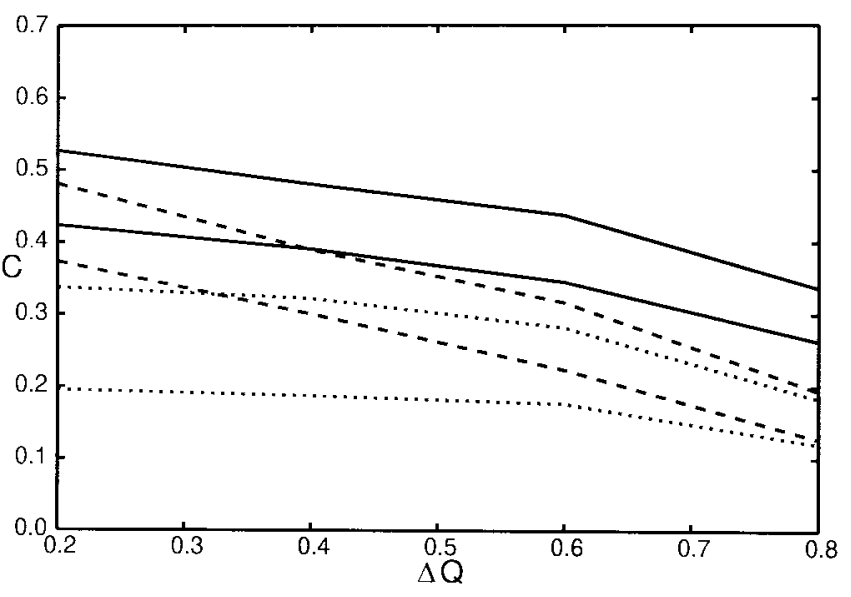

Fig. 7. Correlation coefficient between the true and estimated images for the $\operatorname{MMSE}(-), \operatorname{MAP} 1(---)$ and MAP2 $(\ldots \ldots)$ estimates of $\varrho^{Q}(\mathbf{r})$, as a function of $\Delta Q$, for $S_{\max }=2$ (upper traces), and $S_{\max }=4$ (lower traces).

when there are fewer data $I_{j}$. This effect is referred to as "model bias" by crystallographers and is addressed in detail in a separate paper [28]. Referring to Fig. 9 ( $\Delta Q=$ 


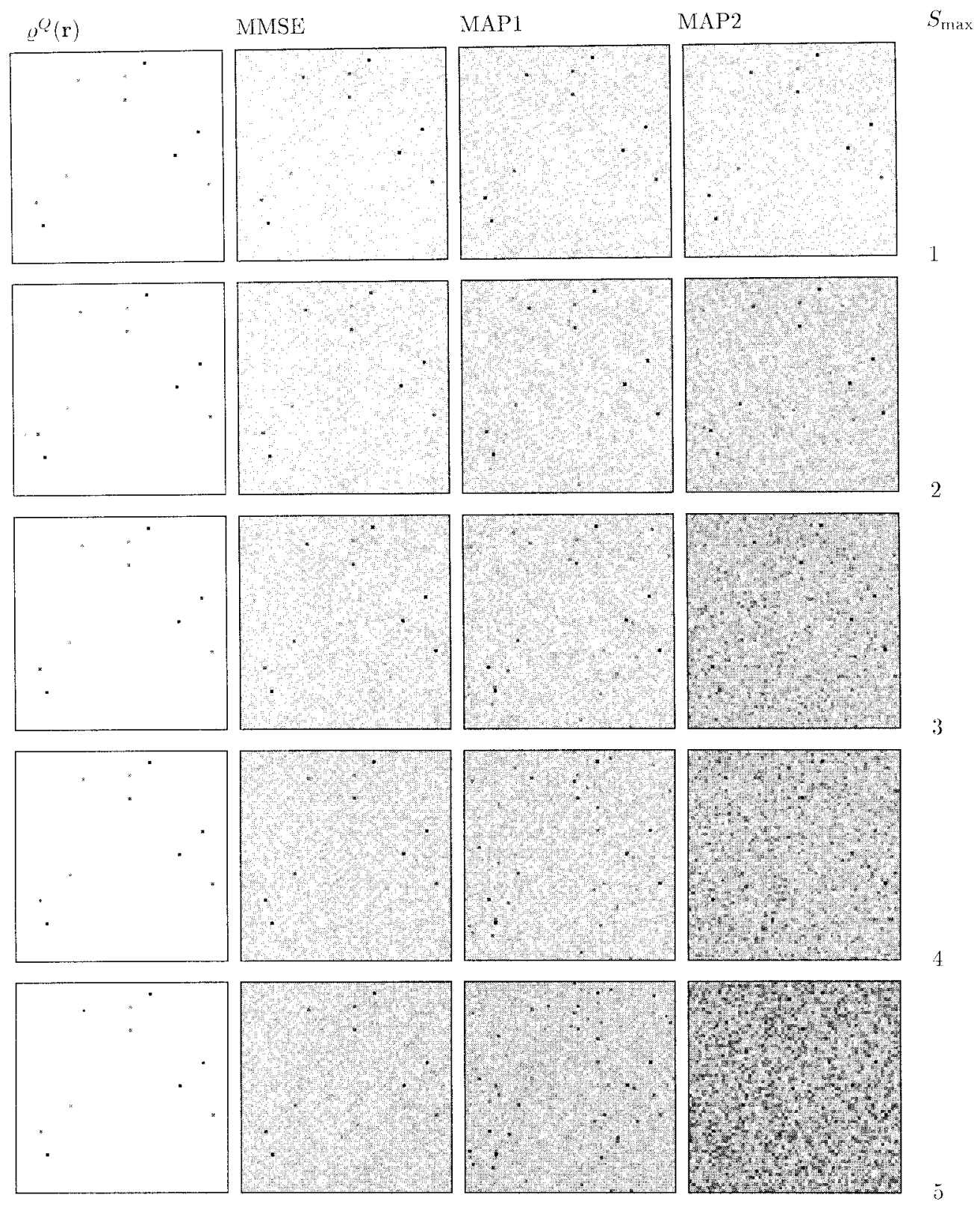

Fig. 8. True image, $\varrho^{Q}(\mathbf{r})$ and the MMSE, MAP1, and MAP2 reconstructions for $\Delta Q=0.2$, and $S_{\max }=1,2,3,4$, and 5 .

0.4) shows that the MMSE estimate is still good for all values of $S_{\max }$ with little evidence of any strong spurious peaks, although the background noise is higher. Both MAP estimates exhibit significant spurious peaks for $S_{\max }>1$. For $\Delta Q=0.6$ (Fig. 10), the MMSE estimate shows most of the missing peaks for the smaller values of $S_{\max }$, although the quality deteriorates for larger $S_{\max }$ where the high background results in little contrast and the appearance of larger spurious peaks. The MAP1 and MAP2 estimates for small $S_{\max }$ show some of the correct peaks although there are many high amplitude spurious peaks. For larger $S_{\max }$, the MAP1 and MAP2 reconstructions are very misleading however, because, although the contrast is quite good, none of the correct peaks are present; all the peaks actually corresponding to peaks of $\varrho^{P}(\mathrm{r})$. Overall, the reconstructed images clearly indicate the superiority of the MMSE reconstructions over the other estimates.

\section{DISCUSSION}

The problem of completing a partially determined structure in x-ray fiber diffraction analysis has been posed as a Bayesian image reconstruction problem and the atomicity property used to define a prior for the image. Currently used heuristic estimators have been shown to correspond to the maxima of certain posterior densities. An analytical expression for the Bayesian MMSE estimate of the image has been derived. The form of the MMSE estimate is a weight times the MAP1 estimate that incorporates all the information on the partial structure. The weights are a generalization of the special case that occurs when the individual structure factor amplitudes 


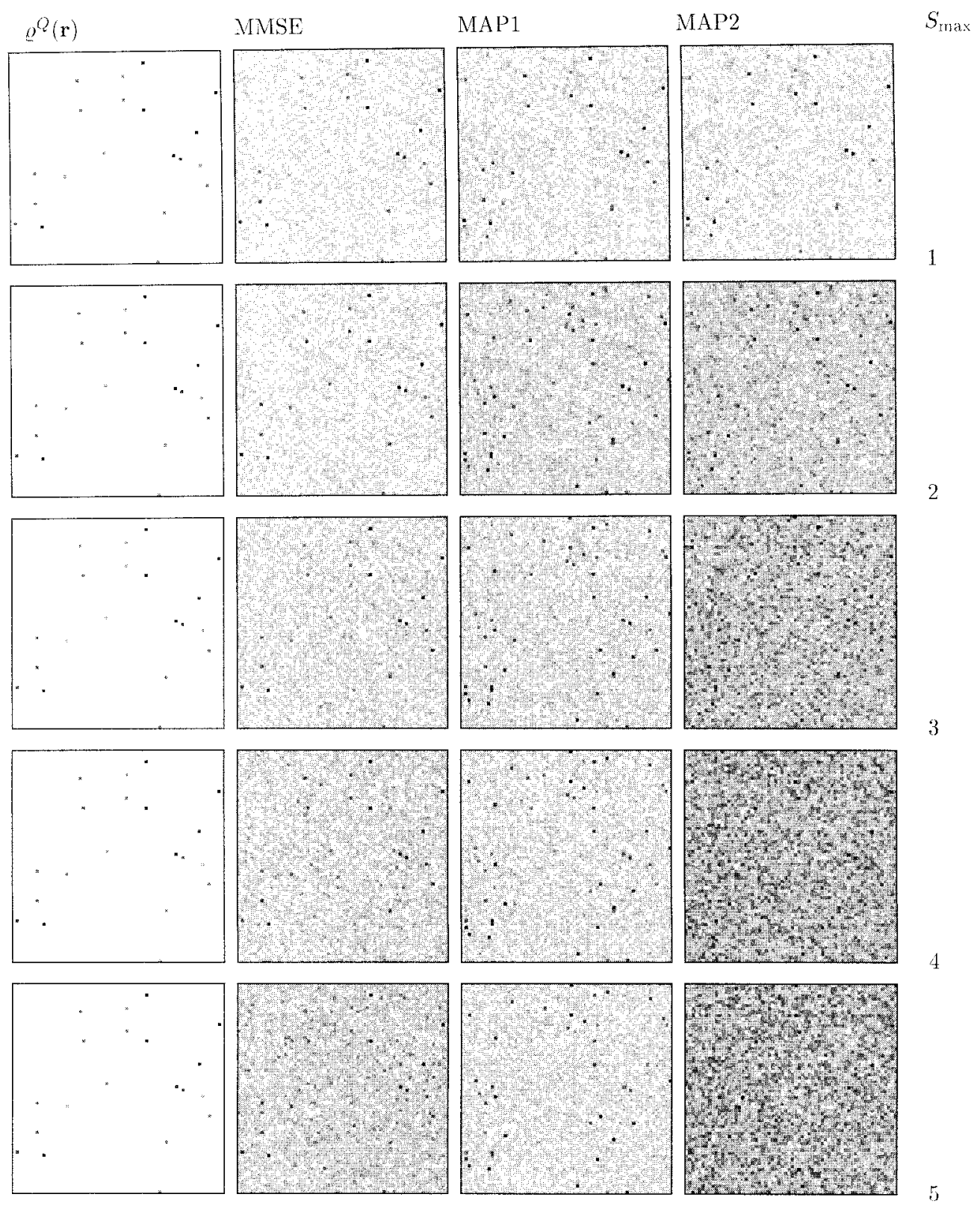

Fig. 9. True image, $\varrho^{Q}(\mathbf{r})$ and the MMSE, MAP1, and MAP2 reconstructions for $\Delta Q=0.4$, and $S_{\max }=1,2,3,4$, and 5 .

can be measured (single crystal diffraction) [2]. Simulations, including parameter estimation, show that the MMSE estimate performs significantly better than the MAP estimates, both quantitatively and qualitatively, particularly when there is little data. This is particularly important in fiber diffraction analysis where the cylindrical averaging can substantially reduce the number of available data.

A number of extensions to this work are possible to further extend its practical application. In a real experiment, both the partial structure and the data have errors associated with them. The effect of inaccurate information can be incorporated into the Bayesian formulation to further improve the performance of the MMSE estimate [29]. The electron density function often contains symmetries that lead to restrictions on and/or relationships between certain structure factors that contribute to the individual intensity data. The simplest case is a "centric" structure, or "space group $\mathrm{P} \overline{1}$," in which case, $n_{j}=\left|\mathcal{S}_{j}\right|$ (rather than $2\left|\mathcal{S}_{j}\right|$ ) and the variance in (17) and the posterior (28) is $\Sigma_{Q}$ (rather than $\Sigma_{Q} / 2$ ), and with these changes the analysis remains the same as presented above. However, the general symmetry case leads to more complicated distributions for the data that result in more complicated estimators. We are currently investigating this case [18], [19]. Finally, in some important fiber diffraction problems, the molecules are oriented parallel and randomly rotated, but do not form crystallites [10], [12]. The measured data are then sums of the squared amplitudes of the Fourier-Bessel transform of the electron density function of the molecule [10]. Completion of partial structures in this case, therefore, requires a slightly different formalism. 

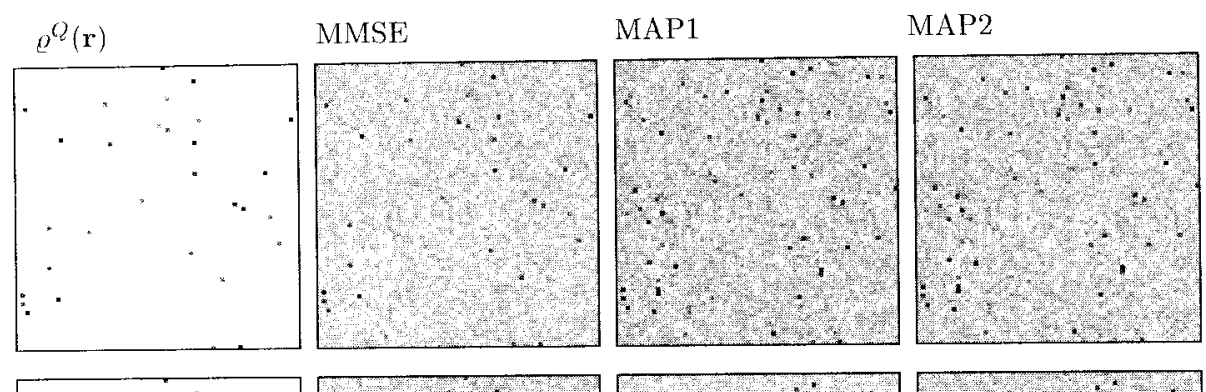

$S_{\max }$
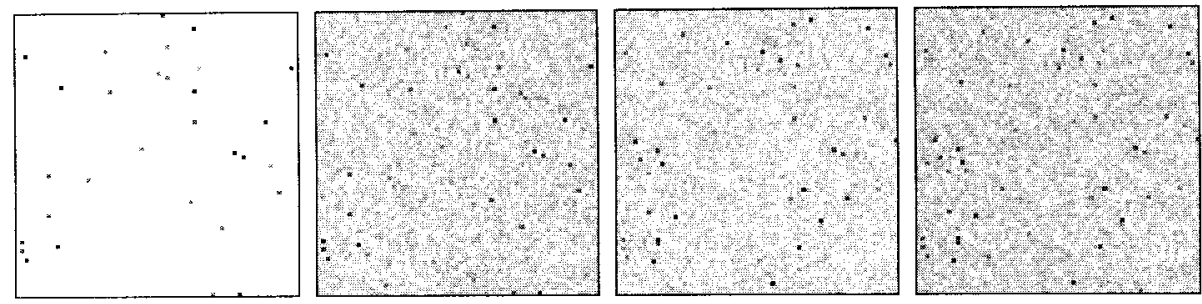

1
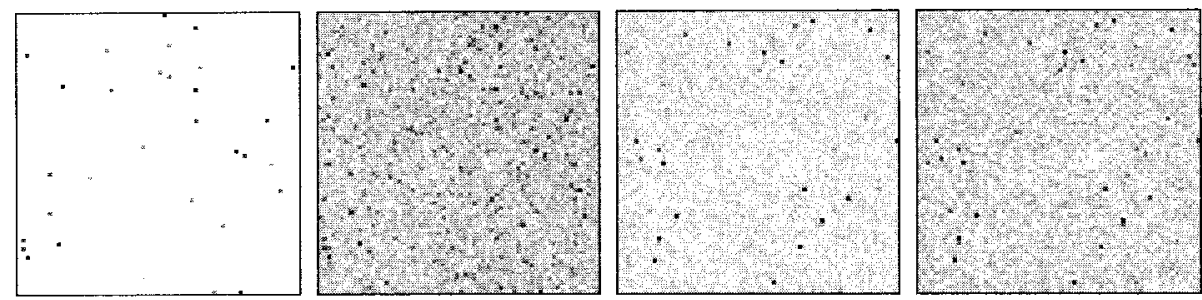

2
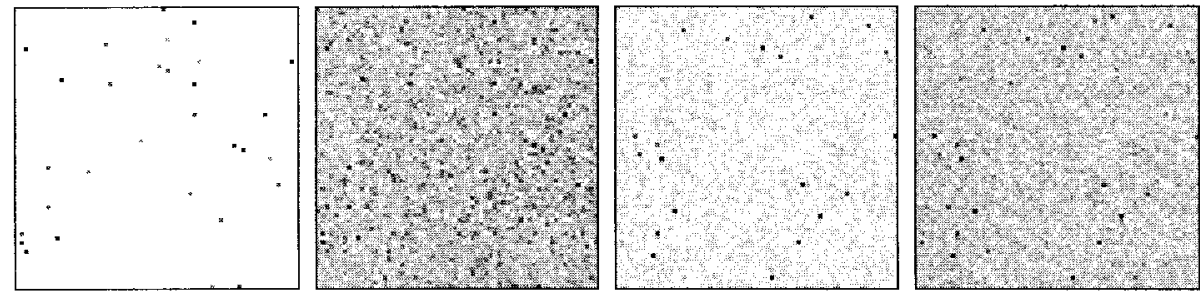

3
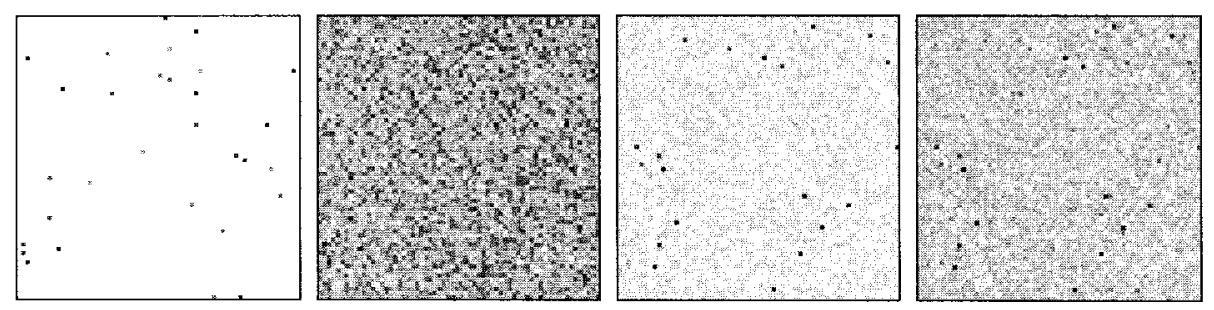

Fig. 10. True image, $\varrho^{Q}(\mathbf{r})$ and the MMSE, MAP1, and MAP2 reconstructions for $\Delta Q=0.6$, and $S_{\max }=1,2,3,4$, and 5 .

\section{APPENDIX A \\ HYPER-SPHERICAL POLAR COORDINATE SYSTEM}

A hyper-spherical polar coordinate system represents a point in an $n$-dimensional space by its radius (Euclidean norm) and $(n-1)$ polar angles $\alpha_{i}$ (hyper-phase $\Omega$ ). The transformation from a Cartesian system $\left[Y_{1}, Y_{2}, \cdots, Y_{n}\right]^{\dagger}$ to a hyper-spherical polar coordinate system $\left[\|\mathbf{Y}\|, \alpha_{1}, \cdots, \alpha_{n-1}\right]^{\dagger}$, or $[\|\mathbf{Y}\|, \Omega]^{\dagger}$, is [30]

$$
\begin{aligned}
\|\mathbf{Y}\| & =\left(\sum_{i} Y_{i}^{2}\right)^{1 / 2} \\
\cos \alpha_{k} & =\frac{Y_{n+1-k}}{\|\mathbf{Y}\| \prod_{i=1}^{k-1} \sin \alpha_{i}} \text { for } k=1 \cdots n-1
\end{aligned}
$$

where $\|\mathbf{Y}\| \geq 0,0 \leq \alpha_{i}<\pi$ for $1<i<n-2$, and $0 \leq \alpha_{n-1}<2 \pi$. The volume element transforms as

$$
\begin{aligned}
d Y_{1} \cdots d Y_{n} & =\|\mathbf{Y}\|^{n-1} d\|\mathbf{Y}\| d \Omega \\
& =\|\mathbf{Y}\|^{n-1} d\|\mathbf{Y}\| \prod_{i=1}^{n-1} \sin ^{n-i-1} \alpha_{i} d \alpha_{i}
\end{aligned}
$$

\section{APPENDIX B \\ MMSE ESTIMATE}

The relationship between the MMSE estimate and the posterior mean, although fairly well known [22], is outlined here. The MMSE estimate minimizes the Bayes risk $R$, the expectation of the loss function $\|\mathbf{Y}-\hat{\mathbf{Y}}\|^{2}$ with respect to the joint density function of the estimated and true images. In the 
absence of errors in the observed intensities, the risk is

$$
R=\prod_{\forall j}\left[\int\left\|\mathbf{y}-\hat{\mathbf{Y}}\left(I_{j}\right)\right\|^{2} P_{\mathbf{Y} \mid I_{j}}(\mathbf{y}) P_{I_{j}}(\cdot) d \mathbf{y}\right] .
$$

The integrals for individual $j, R_{j}$, in (52), are independent and hence may be minimized separately, so that, for the datum $I_{j}$,

$$
\begin{aligned}
\hat{\mathbf{Y}}^{\mathrm{MMSE}} & =\arg \min _{\hat{\mathbf{Y}}} R_{j} \\
& =\arg \min _{\hat{\mathbf{Y}}} \int\left\|\mathbf{y}-\hat{\mathbf{Y}}\left(I_{j}\right)\right\|^{2} P_{\mathbf{Y} \mid I_{j}}(\mathbf{y}) P_{I_{j}}(\cdot) d \mathbf{y} .
\end{aligned}
$$

Since

$$
\left\|\mathbf{y}-\hat{\mathbf{Y}}\left(I_{j}\right)\right\|^{2}=\sum_{i=1}^{n}\left(y_{i}^{2}+\hat{Y}_{i}^{2}-2 y_{i} \hat{Y}_{i}\right)
$$

the minimum in (53) is given by $\partial R_{j} / \partial \hat{Y}_{i}=0$, i.e.

$$
\int\left(\hat{Y}_{i}^{\mathrm{MMSE}}-y_{i}\right) P_{\mathbf{Y} \mid I_{j}}(\mathbf{y}) d \mathbf{y}=0, \quad \forall i
$$

so that

$$
\hat{\mathbf{Y}}^{\mathrm{MMSE}}=\int \mathbf{y} P_{\mathbf{Y} \mid I_{j}}(\mathbf{y}) d \mathbf{y}
$$

i.e., $\hat{\mathbf{Y}}^{\mathrm{MMSE}}$ is the posterior mean of $\mathbf{Y}$.

\section{APPENDIX C}

\section{Evaluation of the Posterior Mean}

The integrals in (29) and (43) are evaluated here.

We treat (43) first. From (50), it follows that

$$
\begin{gathered}
Y_{i}=\|\mathbf{Y}\| \cos \alpha_{n-i+1} \sin \alpha_{1} \cdots \sin \alpha_{n-i}, \\
i=1, \cdots, n
\end{gathered}
$$

where we define $\alpha_{n}=0$. Expanding (43) using (30), (51), and (57), and rotating the Cartesian system so that $Y_{n}$ is along $\boldsymbol{\Theta}$, gives

$$
\begin{aligned}
\hat{Y}_{i}^{\mathrm{MMSE}}= & Z^{-1} \int_{0}^{2 \pi} \int_{0}^{\pi} \cdots \int_{0}^{\pi} \int_{0}^{\infty}\|\mathbf{y}\| \\
& \cdot \cos \alpha_{n-i+1}\left(\prod_{k=1}^{n-i} \sin \alpha_{k}\right) \\
& \cdot \exp \left(-\left[\|\mathbf{y}\|^{2}+\|\boldsymbol{\Theta}\|^{2}-2\|\mathbf{y}\|\|\boldsymbol{\Theta}\| \cos \alpha_{1}\right] / \Sigma_{Q}\right) \\
& \cdot \delta\left(I_{j}-\|\mathbf{y}\|^{2}\right)\|\mathbf{y}\|^{n-1} d\|\mathbf{y}\| \\
& \cdot \prod_{k=1}^{n-1} \sin ^{n-k-1} \alpha_{k} d \alpha_{k} .
\end{aligned}
$$

The integral over $\|\mathbf{y}\|$ collapses due to the delta function. For $i \neq n$, (58) contains integrals of the form

$$
\begin{aligned}
& \int_{0}^{\pi} \cos \alpha_{n-i+1} \sin ^{i-2} \alpha_{n-i+1} d \alpha_{n-i+1} \\
& \text { for } i \geq 2 \\
& \text { or } \quad \int_{0}^{2 \pi} \sin \alpha_{n-1} d \alpha_{n-1} \quad \text { for } i=2
\end{aligned}
$$

each of which yield zero, and hence $\hat{Y}_{i}^{\mathrm{MMSE}}=0$ for $i \neq n$. This implies that $\hat{\mathbf{Y}}^{\mathrm{MMSE}}$ has no components orthogonal to $\Theta$, i.e., $\Omega_{\hat{\mathbf{Y}}}=\Omega_{\Theta}$. Hence $Y_{n}=\|\hat{\mathbf{Y}}\|$. For $i=n$, the integrals over $\alpha_{2}, \cdots, \alpha_{n-1}$ may be separated and evaluated using [30]

$$
\int_{0}^{\pi} \cdots \int_{0}^{\pi} \int_{0}^{2 \pi} \prod_{i=1}^{n-1}\left(\sin \alpha_{i}\right)^{i} d \alpha_{i}=\frac{2 \pi^{n / 2}}{\Gamma(n / 2)}
$$

leading to

$$
\begin{aligned}
\hat{Y}_{n}^{\mathrm{MMSE}}= & \frac{2 \pi^{(n-1) / 2} I_{j}^{1 / 2}}{\Gamma\left(\frac{n-1}{2}\right) Z} \\
& \cdot \int_{0}^{\pi} \exp (\chi \cos \varphi) \cos \varphi(\sin \varphi)^{n-2} d \varphi
\end{aligned}
$$

where

$$
\chi=2 \sqrt{I_{j} I_{j}^{P}} / \Sigma_{Q}
$$

and $\varphi \equiv \alpha_{1}$.

Applying the same coordinate transformation, the normalizing constant (29) may be written as

$$
\begin{aligned}
Z= & \int_{0}^{2 \pi} \int_{0}^{\pi} \cdots \int_{0}^{\pi} \int_{0}^{\infty} \\
& \cdot \exp \left(-\left[\|\mathbf{y}\|^{2}+\|\Theta\|^{2}-2\|\mathbf{y}\|\|\Theta\| \cos \alpha_{1}\right] / \Sigma_{Q}\right) \\
& \cdot \delta\left(I_{j}-\|\mathbf{y}\|^{2}\right)\|\mathbf{y}\|^{n-1} d\|\mathbf{y}\| \\
& \cdot \prod_{k=1}^{n-1} \sin ^{n-k-1} \alpha_{k} d \alpha_{k} .
\end{aligned}
$$

Equation (63) can be simplified in a similar fashion to (58) as above, giving

$$
Z=\frac{2 \pi^{(n-1) / 2}}{\Gamma\left(\frac{n-1}{2}\right)} \int_{0}^{\pi} \exp (\chi \cos \varphi)(\sin \varphi)^{n-2} d \varphi .
$$

Combining (61) and (64) gives

$$
\hat{Y}_{n}^{\mathrm{MMSE}}=\frac{I_{j}^{1 / 2} \int_{0}^{\pi} \exp (\chi \cos \varphi) \sin ^{n-2} \varphi \cos \varphi d \varphi}{\int_{0}^{\pi} \exp (\chi \cos \varphi) \sin ^{n-2} \varphi d \varphi} .
$$

The integral form of the modified Bessel function [31]

$$
I_{\nu}(z)=\frac{(z / 2)^{\nu}}{\pi^{1 / 2} \Gamma(\nu+1 / 2)} \int_{0}^{\pi} \exp (z \cos \zeta) \sin ^{2 \nu} \zeta d \zeta
$$

can be integrated once by parts and used with (66) to show that

$$
\begin{aligned}
I_{\nu+1}(z)= & \frac{(z / 2)^{\nu}}{\pi^{1 / 2} \Gamma(\nu+1 / 2)} \\
& \cdot \int_{0}^{\pi} \exp (z \cos \zeta) \sin ^{2 \nu} \zeta \cos \zeta d \zeta .
\end{aligned}
$$

Substituting (66) and (67) into (65) to gives

$$
\hat{Y}_{n}^{\mathrm{MMSE}}=\sqrt{I_{j}} \frac{I_{n / 2}(\chi)}{I_{n / 2-1}(\chi)} .
$$




\section{REFERENCES}

[1] R. P. Millane, "Phase retrieval in crystallography and optics," J. Opt. Soc. Amer. A., vol. 7, pp. 394-411, Mar. 1990.

[2] J. Drenth, Principles of Protein X-ray Crystallography. Berlin, Germany: Springer-Verlag, 1994.

[3] R. H. T. Bates and M. J. McDonnell, Image Restoration and Reconstruction. Oxford, U.K.: Oxford Univ. Press, 1986.

[4] M. H. Hayes, "The reconstruction of a multidimensional sequence from the phase or magnitude of its Fourier transform," IEEE Trans. Acoust., Speech, Signal Processing, vol. ASSP-30, pp. 140-154, Apr. 1982.

[5] R. G. Lane, W. R. Fright, and R. H. T. Bates, "Direct phase retrieval," IEEE Trans. Acoust., Speech, Signal Processing, vol. ASSP-35, pp. 520-526, Apr. 1987.

[6] J. C. Dainty and J. R. Fienup, "Phase retrieval and image reconstruction for astronomy," in Image Recovery: Theory and Application, H. Stark, Ed. New York: Academic, 1987, pp. 231-275.

[7] A. D. French and K. H. Gardner, Eds., Fiber Diffraction Methods. Washington, DC: Amer. Chem. Soc., 1980.

[8] R. P. Millane, "Structure determination by x-ray fiber diffraction," in Crystallographic Computing 4: Techniques and New Technologies, N. W. Isaacs and M. R. Taylor, Eds. Oxford, U.K.: Oxford Univ. Press, 1988, pp. 169-186.

[9] R. P. Millane, "Image reconstruction from cylindrically averaged diffraction intensities," in Digital Image Recovery and Synthesis II, P. S. Idell, Ed., Proc. SPIE, vol. 2029, pp. 137-143, 1993.

[10] K. Namba and G. Stubbs, "Difference Fourier synthesis in fiber diffraction," Acta Crystallogr., vol. A43, pp. 533-539, July 1987.

[11] R. P. Millane, A. K. Mitra, and S. Arnott, "Chondroitin 4-sulfate: Comparison of the structures of potassium and sodium salts," J. Molec. Biol., vol. 169, pp. 903-920, 1983.

[12] K. Namba, R. Pattanayek, and G. Stubbs, "Visualization of proteinnucleic acid interactions in a virus. Refined structure of intact tobacco mosaic virus at $1.9 \AA$ resolution by x-ray fiber diffraction," J. Molec. Biol., vol. 208, pp. 307-325, July 1989.

[13] S. Baskaran and R. P. Millane, "Bayesian image reconstruction from partial image and spectral amplitude data," in Image Reconstr. Restor. II, T. J. Schulz, Ed., Proc. SPIE, vol. 3170, pp. 227-237, 1997.

[14] G. Bricogne, "A multisolution method of phase determination by combined maximization of entropy and likelihood. III. Extension to powder diffraction data," Acta Crystallogr., vol. A47, pp. 803-829, Nov. 1991

[15] S. Arnott, M. H. F. Wilkins, W. Fuller, and R. Landgridge, "Molecular and crystal structures of double-helical RNA III. An 11-fold molecular model and comparison of the agreement between the observed and calculated three-dimensional diffraction data for 10- and 11-fold models," J. Molec. Biol., vol. 27, pp. 535-548, 1967.

[16] A. J. C. Wilson, "The probability distribution of x-ray intensities," Acta Crystallogr., vol. 2, pp. 318-321, 1949.

[17] M. Woolfson and F. Hai-Fu, Physical and Non-Physical Methods of Solving Crystal Structures. Cambridge, U.K.: Cambridge Univ. Press, 1995.

[18] S. Baskaran and R. P. Millane, "Bayesian image reconstruction with a hyperellipsoidal posterior in x-ray fiber diffraction," in Bayesian Inference for Inv. Prob., A. Mohammad-Djafari, Ed., Proc. SPIE, vol. 3459, pp. 29-38, 1998.

[19] _ in preparation, 1999.

[20] G. Stubbs, "The probability distributions of x-ray intensities in fiber diffraction: Largest likely values of fiber diffraction R factors," Acta Crystallogr., vol. A45, pp. 254-258, Mar. 1989.

[21] R. P. Millane, "Intensity distributions in fiber diffraction," Acta Crystallogr., vol. A46, pp. 552-559, July 1990.

[22] A. Papoulis, Probability, Random Variables and Stochastic Processes. New York: McGraw-Hill, 1991.

[23] M. M. Woolfson, "An improvement on the 'heavy-atom' method of solving crystal structures," Acta Crystallogr., vol. 9, pp. 804-810, 1956.
[24] G. A. Sim, "A note on the heavy-atom method," Acta Crystallogr., vol. 13 , pp. 511-512, 1960.

[25] R. J. Read, "Improved Fourier coefficients for maps using phases from partial structures with errors," Acta Crystallogr., vol. A42, pp. 140-149, Mar. 1986.

[26] R Henderson and J. K. Moffat, "The difference Fourier technique in protein crystallography: Errors and their treatment," Acta Crystallogr., vol. B27, pp. 1414-1420, 1971.

[27] P. E. Nixon and A. C. T. North, "Crystallographic relationships between human and hen-egg lysozymes. II. Weighting of electron density maps phased from incomplete model structure and comparison with map obtained by isomorphous replacement.," Acta Crystallogr., vol. A32, pp. $325-333,1976$

[28] S. Baskaran and R. P. Millane, "Model bias in Bayesian image reconstruction from x-ray fiber diffraction data," J. Opt. Soc. Amer. A., vol. 16, pp. 236-245, Feb. 1999.

[29] _ "An improved prior for image reconstruction in x-ray fiber diffraction," in Proc. IEEE Int. Conf. Image Processing, Chicago, IL, Oct. 4-7, 1998 (on CDROM: Focus Interactive Technol., p. WA9.01).

[30] F. Natterer, The Mathematics of Computerized Tomography. New York: Wiley, 1986.

[31] M. Abramowitz and I. A. Stegun, Eds., Handbook of Mathematical Functions. New York: Dover, 1972, p. 376.

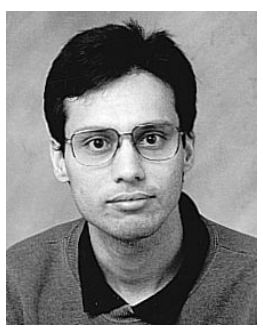

Shyamsunder Baskaran (S'98) was born in Tamil Nadu, India, in 1972. He received the B.S. degree in metallurgical engineering from the Indian Institute of Technology, Madras (now Chennai), in 1993. He is currently pursuing the Ph.D. degree Computational Science and Engineering Program, Purdue University, West Lafayette, IN. His research interests include inverse problems, phase retrieval, and crystallographic algorithms.

Mr. Baskaran received a National Talent Scholarship from the government of India in 1987.

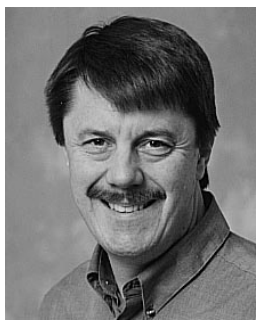

Rick P. Millane (M'82-SM'94) received the B.E. and $\mathrm{Ph} . \mathrm{D}$. degrees in electrical engineering from the University of Canterbury, Christchurch, New Zealand, in 1975 and 1981.

$\mathrm{He}$ was a telecommunications engineer with the New Zealand Post Office (now Telecom New Zealand) for two years. He came to Purdue University, West Lafayette, IN, in 1982, where he is now a Professor in the Whistler Center for Carbohydrate Research and the Computational Science and Engineering Program. He has held visiting appointments in the Department of Electrical and Electronic Engineering, University of Canterbury, New Zealand (as an Erskine Fellow), and in the Marine Physical Laboratory, Scripps Institution of Oceanography, La Jolla, CA. His research activities involve inverse problems, phase retrieval, image reconstruction and diffraction, with applications in x-ray crystallography, polymer structure, optical tomography, and ocean acoustics.

Dr. Millane has served as Chairman of the ACA Special Interest Group on Fiber Diffraction, and as Technical Chairman of the OSA Topical Meeting on Signal Recovery and Synthesis in 1998. He has been on program committees for IEEE-APS, OSA, SPIE, IUCr, and ACA meetings. He is a member of the Optical Society of America and the American Crystallographic Association. 